\title{
Cocovering And Set Forcing
}

\author{
M.C. Stanley
}

November 2003

1. Introduction . . . . . . . . . . . . . . . . . . . . . . . . 1

2. Cocovering, covering, and set genericity with $\mathrm{GCH}$ assumptions . . . . . 5

2.1. $\kappa$-cocovering . . . . . . . . . . . . . . . . . . . . 5

2.2. $\kappa$-covering . . . . . . . . . . . . . . . . . . . . . . 5

2.3. Set forcing, covering, and cocovering . . . . . . . . . . . . . . . 7

3. $\pitchfork_{\lambda,<\kappa}$ and set genericity without GCH assumptions . . . . . . . . . . 9

4. Applications . . . . . . . . . . . . . . . . . . . . . . . . . . . 12

4.1. Set generic outer models . . . . . . . . . . . . . . . . . . . . . 12

4.2. Supercompact cardinals . . . . . . . . . . . . . . . . . . . 15

5. Superdensity reduction . . . . . . . . . . . . . . . . . . . . . 18

6. Iterated reduction . . . . . . . . . . . . . . . . . . . . . . . 21

7. The factor lemma . . . . . . . . . . . . . . . . . . . . . . . . . 24

8. The set forcing theorem . . . . . . . . . . . . . . . . . . . . . . 28

9. References . . . . . . . . . . . . . . . . . . . . . 30

\section{Introduction}

Let $V[G]$ be the result of adding a Cohen real and a Cohen subset of $\omega_{2}$ to a model $V$ of the GCH. Every infinite cardinal gets a new subset in $V[G]$, namely, the Cohen real. Yet, in some sense, only $\omega$ and $\omega_{2}$ get new subsets. One way to capture this is to say that a sort of dual to Covering holds between $V$ and $V[G]$, namely, " $\kappa$-cocovering" for $\kappa$ other than $\omega$ and $\omega_{2}$.

We need some definitions.

If $\kappa$ is a regular cardinal in $V$, say that $\boldsymbol{\kappa}$-cocovering holds between $V$ and the outer model $W$ if, given any unbounded $a \subseteq \kappa$ that lies in $W$, there exists $b \in V$ such that $b \subseteq a$ and $b$ is unbounded in $\kappa$.

If $V$ is a standard transitive model of ZFC, say that $W \supseteq V$ is an outer model of $V$ if $W$ is also a standard transitive model of ZFC and $V \cap \mathrm{OR}=W \cap \mathrm{OR}$. In this paper we are only concerned with outer models such that $(W ; V)$ satisfies ZFC (in a language with a predicate symbol for $V$ ).

Ultimately, ZFC is our metatheory; talk of models can be understood as talk of set, or even countable set models. The reader will note that our results usually can be paraphrased in more traditional terminology, perhaps at the cost of some generality.

This paper proves a sort of converse to the observation with which we began. The general case is not quite so simple as this example suggests. We show that if $W$ is any outer model of $V$, and sufficient cocovering (and maybe a little covering) hold around $\kappa$, then every subset of $\kappa$ that lies in $W$ is set generic over $V$ for a forcing of $V$-cardinality less than $\kappa$.

Research supported by N.S.F. Grant DMS 9505157.

This is version 1.101 . 


\section{INTRODUCTION}

To be precise, let us begin with a special case in which we assume a little of the GCH in the inner model $V$. Corollary 2.12 draws the following corollary of our main theorem.

Theorem. Assume that

- in $V: \kappa$ is regular, $\kappa^{<\kappa}=\kappa$, and $2^{\kappa}=\kappa^{+}$. Also assume that

- both $\kappa$ and $\kappa^{+}$-cocovering hold between $V$ and $W$.

Then every subset of $\kappa^{+}$that lies in $W$ is set generic over $V$ for a forcing of $V$ cardinality less than $\kappa$.

In light of the example with which we began, requiring both $\kappa$ and $\kappa^{+}$-cocovering is a surprise. Example 2.15 shows that this is necessary in general.

The author's interest in this topic was sparked by considering alternative strategies for coding the universe. All known proofs of Jensen's Coding Theorem use variants of almost disjoint coding. A generic real codes a subset of $\omega_{1}$; that subset of $\omega_{1}$ codes a subset of $\omega_{2}$; and so forth. Coding requirements (at least) at limits eventually overlap. Substantial technical machinery is necessary to handle the project. Could there be an easier way?

One informal idea is this: Rather than proceeding cardinal-by-cardinal, code across bigger gaps in the ordinals, say using homogeneous sets for partitions of some sort in place of almost disjoint coding. The author's opinion is that the theorem stated above implies that such a program is unlikely succeed. Over a model of the GCH (for example, $V=L$ ), a real that does not add "genuinely new" subsets to some $V$-regular cardinal and its $V$-successor is set-generic.

Something like its cocovering hypotheses is necessary for this theorem. On the other hand, the cardinal arithmetic assumptions on $V$ appear spurious. To eliminate these, we need a more devilish form of cocovering:

$\left(\pitchfork_{\lambda,<\kappa}\right)$

For each $F:[\lambda]^{<\kappa} \rightarrow \lambda$ in $W$, there exists $f \in V$ such that $f \subseteq F$ and $\bigcup \operatorname{dom}(f)=\lambda$.

We might remark that $\pitchfork_{\lambda,<\kappa}$ is just a two-variable generalization of $\lambda$-cocovering. Another way to state $\lambda$-cocovering is, given $F: \lambda \rightarrow \lambda$ that lies in $W$, there exists $f \in V$ such that $f \subseteq F$ and $\bigcup \operatorname{dom}(f)=\lambda$. Indeed, for $V$-regular $\kappa, \pitchfork_{\kappa,<\kappa}$ is equivalent to $\kappa$-cocovering (see Lemma 3.4).

Like $\lambda$-cocovering, if $G$ is generic for a forcing of $V$-cardinality less than $\kappa$, then $\pitchfork_{\lambda,<\kappa}$ holds between $V$ and $W=V[G]$, for all $\lambda \geqslant \kappa$. (See Lemma 3.2.)

Using $\pitchfork_{\lambda,<\kappa}$ in place of cocovering, we get for example (Corollary 3.7):

Theorem. In $V$, let $\kappa$ be a regular uncountable cardinal and set $\lambda=2^{\kappa}$. Assume that there are no limit cardinals (of $V$ ) between $\kappa$ and $\lambda$. If $\pitchfork_{\lambda,<\kappa}$ holds, then every subset of $\kappa^{+}$in $W$ is set generic over $V$ for a forcing of $V$-cardinality less than $\kappa$.

If we allow more characters in our story, we can eliminate the (again spurious) hypothesis that $\left(2^{\kappa}\right)^{V}$ is not much larger than $\kappa$. In full generality, the main theorem of this paper (Theorem 8.1) is 


\section{INTRODUCTION}

Set Forcing Theorem. In $V$, let $\kappa, \mu$, and $\lambda$ be cardinals such that

- $\omega_{1} \leqslant \kappa<\mu \leqslant \lambda$,

- $\kappa$ is regular,

- $\operatorname{cf}(\mu)>\kappa$, and

- $\lambda^{<\mu}=\lambda$.

Assume that

- $<\kappa$-covering for subsets of $\lambda$ and

- $\pitchfork_{\lambda,<\kappa}$

hold between $V$ and $W$. Then every subset of $\mu$ in $W$ is set generic over $V$ for a forcing of $V$-cardinality less than $\kappa$.

$<\kappa$-covering for subsets of $\lambda$ is the requirement that, given any $a \subseteq \lambda$ that has $W$-cardinality less than $\kappa$, there exists $b \in V$ such that $b \supseteq a$ and $b$ has $V$-cardinality less than $\kappa$. If there are no limit cardinals between $\kappa$ and $\lambda$, then $<\kappa$-covering for subsets of $\lambda$ is a consequence of $\pitchfork_{\lambda,<\kappa}$. (See Lemmas 3.3 and 3.8.)

Like $\lambda$-cocovering and $\pitchfork_{\lambda,<\kappa}$, for $\lambda \geqslant \kappa,<\kappa$-covering is preserved by forcing of size less than $\kappa$.

The Set Forcing Theorem is a corollary of a factoring lemma for class forcing (Lemma 7.1). But the Set Forcing Theorem applies to arbitrary outer models of $V$. How is this possible?

If $\mathbb{P}$ is a $V$-amenable partial ordering, say that a filter $G \subseteq \mathbb{P}$ is internally generic if $G$ decides every set $d \in V$ of conditions. That is, there exists $p \in G$ such that either $p \in d$ or $p$ is incompatible with every element of $d$. If $\mathbb{P} \in V$, then this is equivalent to ordinary (set) genericity. However in the case of class forcing, this sort of genericity is too weak to be of much interest. (See $[\mathbf{S}]$ for a discussion of levels of class genericity.)

It is an essentially trivial observation that any outer model of $V$ is an internally generic extension of $V$ (see Lemma 8.2). A substantial fraction of the effort that goes into proving the Factor Lemma goes into seeing that it applies to forcing even in the weak sense of internal genericity. The point is that even internal genericity over a set-sized factor amounts to ordinary set genericity.

If $V$ is "sufficiently non-minimal" as an inner model of $W$, then $W$ is a generic extension of $V$ in a better sense: There exists a $V$-amenable class partial ordering $\mathbb{P}$ and a filter $G$ such that $W=V[G]$ and $G$ is "generic with respect to the language of set theory." This is the main theorem of $[\mathbf{S}]$. No use is made of it in this paper, but the Factor Lemma is proved in a form that applies to this forcing, as well as to forcing in larger languages and to set forcing.

In $\S 4$ we pursue two sorts of applications of the Set Forcing Theorem. One is to give a combinatorial characterization of set-forcing extensions. This was first done in the early 1970s by Vopěnka and Hájek and, in a different way, later by Bukovský (see [B]). These are discussed in $\S 4.1$. The former result is a corollary of our characterization (see Lemma 4.7); the latter is of a different nature.

Let Jensen $\kappa$-covering be the following principle: If $a \in W$ is a set of ordinals, then there exists $b \in V$ such that $b \supseteq a$ and $|b|^{W} \leqslant \max \left(\kappa,|a|^{W}\right)$. 


\section{INTRODUCTION}

In this terminology, Jensen's Covering Lemma is that if $W$ satisfies " 0 " does not exist," then Jensen $\omega_{1}^{W}$-covering holds.

Theorem 4.3 characterizes set-forcing extensions, assuming some of the GCH in $V$.

Theorem. Assume that in $V$ there exist unboundedly many regular $\kappa$ such that $\kappa^{<\kappa}=$ $\kappa$ and $2^{\kappa}=\kappa^{+}$. The following are equivalent:

(a) $W$ is a set generic extension of $V$.

(b) There exists a $W$-cardinal $\nu$ such that

- Jensen $\nu$-covering and

- $\lambda$-cocovering, for $\lambda \geqslant \nu$,

hold between $V$ and $W$.

Using $\pitchfork_{\lambda,<\nu}$, Theorem 4.2 characterizes set forcing extensions without the assumptions on cardinal arithmetic in $V$.

The other application in this paper concerns supercompact cardinals. If $D$ is a normal fine $\kappa$-complete ultrafilter on $\mathcal{P}_{\kappa} \lambda$, let $j_{D}$ be the associated ultrapower embedding. Let $D$ be such an ultrafilter in $V$. In $(W ; V)$ define

$$
\widehat{D}=\left\{A \subseteq \mathcal{P}_{\kappa} \lambda: \exists B \in D B \subseteq A\right\} .
$$

Say that $D$ automatically extends in $W$ if,

- in $W, \widehat{D}$ is a normal fine $\kappa$-complete ultrafilter on $\mathcal{P}_{\kappa} \lambda$ and

- $j_{\widehat{D}} \uparrow V=j_{D}$.

If $W=V[G]$, where $G$ is $\mathbb{P}$-generic over $V$ and $\mathbb{P} \in V_{\kappa}$, then $D$ automatically extends in $W$. Theorem 4.10 proves a converse:

Theorem. In $V$, let $\lambda \geqslant \mu>\kappa$ be uncountable cardinals such that $\kappa$ is regular, $\operatorname{cf}(\mu)>\kappa$, and $\lambda^{<\mu}=\lambda$. If there exists a normal ultrafilter $D \in V$ on $\left(\mathcal{P}_{\kappa} \lambda\right)^{V}$ that automatically extends in $W$, then each subset of $\mu$ that lies in $W$ is $\mathbb{P}$-generic over $V$, for some forcing $\mathbb{P} \in V_{\kappa}$.

If this happens at every $\lambda \geqslant \kappa$, then $W$ is a set-generic extension of $V$ (Theorem 4.11):

Theorem. Suppose that for every $\lambda \geqslant \kappa$ there exists a normal ultrafilter $D \in V$ on $\left(\mathcal{P}_{\kappa} \lambda\right)^{V}$ that automatically extends in $W$. Then there exists a forcing $\mathbb{P} \in V_{\kappa}$ and a filter $G$ such that $G$ is $\mathbb{P}$-generic over $V$ and $W=V[G]$.

Proposition 4.13 shows that the analog of Theorem 4.10 for measures on $\kappa$ is false.

This paper has eight sections. Section 2 develops some useful facts about $\kappa$-covering and $\kappa$-cocovering. Section 3 does the same for $\pitchfork_{\lambda,<\kappa}$. Using this work, Section 4 makes the applications mentioned above. Sections 5-8 prove the Set Forcing Theorem. Section 5 establishes the basic consequence of $\pitchfork_{\lambda,<\kappa}$ used in the proof, namely, "superdensity reduction". Section 6 applies it repeatedly to get a technical Iterated Reduction Lemma aimed at "closing off" in set cardinality the set-complete subalgebra generated by a set of conditions. Section 7 applies this apparatus to prove the Factor Lemma. Section 8 then easily concludes the Set Forcing Lemma from the Factor Lemma.

So far as the author knows, with one exception, the notation in this paper is consistent with $[\mathbf{J}]$. That exception is that $\ll \alpha, \beta »$ is used to indicate Gödel paring. 


\section{Cocovering, covering, and set genericity with GCH assumptions}

In this section, adopt the convention that $V$ is a standard transitive model of ZFC and that $W$ is an outer model of $V$ such that $(W ; V) \vDash \mathrm{ZFC}$.

\section{1. $\kappa$-cocovering}

Recall from $\S 1$ the following

Definition. Suppose that $\kappa$ is an infinite regular cardinal in the inner model $V$. Say that $\boldsymbol{\kappa}$-cocovering holds between $V$ and $W$ if, given any unbounded $a \subseteq \kappa$ that lies in $W$, there exists an unbounded $b \subseteq a$ that lies in $V$.

The following observation is universally known.

Proposition 2.1. In $V$, let $\kappa$ be regular and let $\mathbb{P}$ be a partial ordering of cardinality less than $\kappa$. If $G$ is $\mathbb{P}$-generic over $V$, then $\kappa$-cocovering holds between $V$ and $V[G]$.

Proof: This is easy to prove directly. Alternatively, it follows from Lemmas 3.1 and 3.3 .

Our definition of " $\kappa$-cocovering" presupposes that $\kappa$ is regular in the inner model $V$. Trivially, we have

Lemma 2.2. If $\kappa$-cocovering holds between $V$ and $W$, then $\kappa$ is also regular in the outer model $W$.

Further evidence that $\kappa$-cocovering is a strong hypothesis is provided by the following two easy lemmas that will be useful later.

Lemma 2.3. If $\omega$-cocovering holds between $V$ and $W$, then $\mathcal{P}^{W}(\omega)=\mathcal{P}^{V}(\omega)$.

Proof: In $V$, fix a bijection $f:[\omega]^{<\omega} \rightarrow \omega$. Let $a \in W$ be a subset of $\omega$. If $b \in V$ is an unbounded subset of $f^{\prime \prime}\{a \cap n: n \in \omega\}$, then $a=\bigcup_{i \in b} f^{-1}(i)$ lies in $V$.

Lemma 2.4. If $\kappa$-cocovering holds between $V$ and $W$, and then $\left(\kappa^{+}\right)^{W} \leqslant\left(2^{\kappa}\right)^{V}$.

Proof: If $\kappa=\omega$, this follows from Lemma 2.3. Assume that $\kappa$ is uncountable in $V$ (hence in $W$ ). For a contradiction, suppose that $\left(2^{\kappa}\right)^{V}<\left(\kappa^{+}\right)^{W}$. In $(W ; V)$, let $C$ be a diagonal intersection of all of the club subsets of $\kappa$ that lie in $V$. Working in $V$, let $D$ be the closure of an unbounded subset of $C$. Then $D$ is eventually contained in every club subset of $\kappa$, which is absurd.

\section{2. $\kappa$-covering}

Definition. If $\kappa$ is a cardinal in the outer model $W$ and $\lambda \geqslant \kappa$, say that $\boldsymbol{\kappa}$-covering for subsets of $\lambda$ holds between $V$ and $W$ if, given any $a \subseteq \lambda$ such that $a \in W$ and $|a|^{W}=\kappa$, there exists $b \in V$ such that $b \supseteq a$ and $|b|^{V}=\kappa$. Say that $\kappa$-covering holds between $V$ and $W$ if $\kappa$-covering for subsets of $\lambda$ holds for all $\lambda \geqslant \kappa$. Let $<\kappa$-covering for subsets of $\lambda$ and $<\kappa$-covering have the same definitions with " $<$ " in place of " $=$ ". 
In general, the requirement that the covering set $b$ has cardinality $\kappa$ in the inner model $V$ is strong. The context of this paper typically softens this in the sense that usually we also assume some cocovering, which implies that relevant $V$-cardinals are $W$-cardinals.

Like Proposition 2.1, another familiar observation is

Proposition 2.5. Let $\kappa$ be regular in $V$ and let $\mathbb{P} \in V$ be a partial ordering of $V$ cardinality less than $\kappa$. If $G$ is $\mathbb{P}$-generic over $V$, then $<\kappa$-covering and $\lambda$-covering, for $\lambda \geqslant \kappa$, hold between $V$ and $V[G]$.

The following two examples show that covering and cocovering are independent phenomena.

Example 2.6. A pair of models such that

(a) $\kappa$-covering holds for all $W$-cardinals $\kappa$, but

(b) $\kappa$-cocovering fails for all $V$-regular $\kappa$.

Let $V$ satisfy the GCH and let $W=V[G]$ be the result of adding a Cohen subset to each $V$-regular $\kappa$ using an Easton support product.

Example 2.7. A pair of models such that

(a) there exists an uncountable $W$-cardinal $\lambda$ such that $\kappa$-covering for subsets of $\lambda$ fails for all $W$-cardinals $\kappa \leqslant \lambda$, but

(b) $\kappa$-cocovering holds for all $V$-regular $\kappa$.

Let $\lambda$ be measurable in $\bar{V}$. Let $y$ be a $\bar{V}$-generic Prikry sequence, and let $x$ consist of every other element of $y$. Set $V=\bar{V}[x]$ and $W=\bar{V}[y]=V[y]$.

Together covering and cocovering are strong indeed. The following lemma will be useful in applications.

Lemma 2.8. Assume that

- $\kappa$-cocovering holds, for all infinite $V$-regular $\kappa$, and

- $\kappa$-covering holds, for all uncountable $W$-cardinals $\kappa$.

Then $V=W$.

The models $V$ and $W$ have the same cardinals and cofinalities under the hypothesis that $\kappa$-cocovering holds for all infinite $V$-regular $\kappa$. Thus, in the presence of the first hypothesis, the second is just that Jensen's Covering Lemma holds between $V$ and $W$. Proof of Lemma 2.8: Proceed by induction on infinite $\kappa$ to see that $\mathcal{P}^{V}(\kappa)=$ $\mathcal{P}^{W}(\kappa)$. This suffices, since $V$ and $W$ are standard transitive models of ZFC with the same ordinals.

If $\kappa=\omega$, then we have already seen that $\omega$-cocovering implies $\mathcal{P}^{V}(\omega)=\mathcal{P}^{W}(\omega)$.

Now suppose that $\kappa>\omega$ is a cardinal and that any bounded subset of $\kappa$ in $W$ lies in $V$. In $V$, fix a one-to-one enumeration $\left\langle x_{i}: i<\lambda\right\rangle$ of all bounded subsets of $\kappa$. Also fix in $V$ an increasing sequence $\left\langle\alpha_{\delta}: \delta<\operatorname{cf}(\kappa)\right\rangle$ cofinal in $\kappa$.

In $W$, fix $x \subseteq \kappa$. We may assume that $x$ is unbounded in $\kappa$. Set

$$
I=\left\{i<\lambda: x_{i}=x \cap \alpha_{\delta}, \text { for some } \delta<\operatorname{cf}(\kappa)\right\} .
$$


Let $\alpha$ be least such that $|I \cap \alpha|=\operatorname{cf}(\kappa)$. Then $\operatorname{cf}(\alpha)=\operatorname{cf}(\kappa)$ and $\bigcup_{i \in I \cap \alpha} x_{i}=x$. Let $J \in V$ be such that $J \supseteq I \cap \alpha$ and $|J| \leqslant \max \left(\operatorname{cf}(\kappa), \omega_{1}\right) \leqslant \kappa$. Let $f \in V$ be a bijection $f:|J| \rightarrow J$ and let $z$ be the inverse image of $I$ under $f$. If $|J|<\kappa$, then $z \in V$ by induction, hence $x=\bigcup_{i \in f " z} x_{i} \in V$. So suppose $|J|=\kappa$. Then $\kappa=\max \left(\operatorname{cf}(\kappa), \omega_{1}\right)$. It follows that $\kappa=\operatorname{cf}(\kappa)$, since otherwise $\kappa=\omega_{1}>\operatorname{cf}(\kappa)$, which is absurd. By $\kappa$ cocovering, let $y \in V$ be a subset of $z$ that is cofinal in $\kappa$. Then $f^{\prime \prime} y$ is cofinal in $I \cap \alpha$. It follows that $x=\bigcup_{i \in f "} y x_{i} \in V$.

The following easy observation is used in the proof of Corollary 2.12.

Lemma 2.9. Assume that $\kappa$ is regular in $W$ Then $\kappa$-covering for subsets of $\lambda$ implies $<\kappa$-covering for subsets of $\lambda$.

Proof: Let $b \subseteq \lambda$ be a set of $W$-cardinality less than $\kappa$. In $W$, extend $b$ to $b^{\prime}$ having cardinality $\kappa$. Let $a^{\prime} \in V$ have $V$-cardinality $\kappa$ and cover $b^{\prime}$. Let $f: a^{\prime} \rightarrow \kappa$ be a bijection in $V$. Because $\kappa$ is regular in $W$, there exists $\delta<\kappa$ such that $f$ " $b \subseteq \delta$. The inverse image of $\delta$ under $f$ is a set in $V$ of $V$-cardinality less than $\kappa$ that covers $b$.

\subsection{Set forcing, covering, and cocovering}

The next two lemmas concern two ways in which forcing can kill cocovering. If $\kappa^{<\kappa}=\kappa$ in $V$, then $\kappa$-covering for subsets of $\left(\kappa^{<\kappa}\right)^{V}$ is trivial. It is a consequence of the first of these lemmas that, in this case, if $\kappa$ is (uniformly) least such that forcing with $\mathbb{P}$ adds a subset to $\kappa$, then $\kappa$-cocovering fails between $V$ and any $\mathbb{P}$-generic extension.

The second lemma is that if $\kappa$ is the uniform density of $\mathbb{P}$, then forcing with $\mathbb{P}$ kills $\kappa$-cocovering.

An example of both is provided by Sacks forcing over a model of $\mathrm{CH}$. The least ordinal (uniformly) that gets a new subset in a Sacks extension is $\omega$; assuming $\mathrm{CH}$, Sacks forcing has uniform density $\omega_{1}$. Neither $\omega$ nor $\omega_{1}$-cocovering holds between $V$ and a Sacks generic extension.

Lemma 2.10. Let $\mathbb{P} \in V$ be a partial ordering and suppose that the $V$-regular cardinal $\kappa$ is uniformly least such that forcing with $\mathbb{P}$ adds a new subset to $\kappa$. Let $G$ be $\mathbb{P}$-generic over $V$. Then at least one of the following fails between $V$ and $V[G]$ :

- $\kappa$-cocovering and

- $\kappa$-covering for subsets of $\left(\kappa^{<\kappa}\right)^{V}$.

Proof: Suppose not. In $V$, let $f:[\kappa]^{<\kappa} \rightarrow \lambda$ be a bijection, where $\lambda=\kappa^{<\kappa}$. Suppose $a \in V[G] \backslash V$ and $a \subseteq \kappa$. Then $a$ is unbounded in $\kappa$ and $a \cap \alpha \in V$, for $\alpha<\kappa$ by our choice of $\kappa$. Using $\kappa$-covering, let $b \in V$ be such that $b \supseteq\{f(a \cap \alpha): \alpha<\kappa\}$ and $|b|^{V}=\kappa$. Let $g \in V$ be a bijection $g: b \rightarrow \kappa$. Then $g$ " $\{f(a \cap \alpha): \alpha<\kappa\}$ is unbounded in $\kappa$. Using $\kappa$-cocovering, let $c \in V$ be an unbounded subset of this subset of $\kappa$. Then $a=\bigcup_{\gamma \in c} f^{-1}\left(g^{-1}(\gamma)\right)$, contradicting that $a \notin V$.

Lemma 2.11. In $V$, let $\kappa$ be regular and let $\mathbb{P}$ be a separative partial ordering of uniform density $\kappa$. If $G$ is $\mathbb{P}$-generic over $V$, then $\kappa$-cocovering fails between $V$ and $V[G]$.

Proof: Work in $V$. Let $\left\langle p_{i}: i<\kappa\right\rangle$ enumerate a dense subset $D$ of $\mathbb{P}$. For convenience, assume that each element of $D$ appears $\kappa$ times in this enumeration. Define 


\subsection{SET FORCING, COVERING, AND COCOVERING}

$\left\langle q_{i}: i<\kappa\right\rangle$ by recursion: Choose $q_{i} \leqslant p_{i}$ such that $q_{i} \in D$ and, for all $j<i$, either $q_{i} \mid q_{j}$ or $q_{i}$ properly extends $q_{j}$. Such a condition $q_{i}$ exists because $\left\{q_{j}: j<i\right\}$ is not dense below $p_{i}$.

Let us make two observations. First, using that each element of $D$ occurs $\kappa$ times in the enumeration $\left\langle p_{i}: i<\kappa\right\rangle$, it is easy to see that $\left\{p \in \mathbb{P}: \exists i \geqslant \beta\left(p \leqslant q_{i}\right)\right\}$ is dense, for each $\beta<\kappa$.

Secondly, if $p \in \mathbb{P}$, we can choose $j$ to be least such that $p_{j} \leqslant p$. Then $q_{j} \leqslant p$. If $i>j$, then either $q_{i} \mid q_{j}$ or $q_{i}$ is a proper extension of $q_{j}$. In either case, $p \nless q_{i}$. Using that $\mathbb{P}$ is separative, it follows that $p \| \check{q}_{i} \in \dot{G}$.

By these two observation, if $G$ is $\mathbb{P}$-generic over $V$, then $a=\left\{i<\kappa: q_{i} \in G\right\}$ witnesses that $\kappa$-cocovering fails between $V$ and $V[G]$.

The following corollary is derived from the main theorem of this paper in $\S 3$. (See the proof just before Lemma 3.6.) That theorem uses a two variable form of cocovering (the principle $\pitchfork_{\lambda,<k}$ stated $\S 1$ ) that allows us to eliminate the hypotheses on cardinal arithmetic in $V$ that appear in the statement of Corollary 2.12.

Corollary 2.12. Let $\kappa$ be regular in $V$ and assume the following:

- $\kappa^{<\kappa}=\kappa$ and $2^{\kappa}=\kappa^{+}$in $V$ and

- both $\kappa$ and $\kappa^{+}$-cocovering hold between $V$ and $W$.

Then every subset of $\kappa^{+}$that lies in $W$ is set generic over $V$ for a partial ordering of $V$-cardinality less than $\kappa$.

Note the following consequence of Corollary 2.12: If in $V$ there are unboundedly many regular $\kappa$ such that $\kappa^{<\kappa}=\kappa$ and $2^{\kappa}=\kappa^{+}$, and if $\lambda$-cocovering holds between $V$ and $W$, for all sufficiently large $\lambda$, then every element of $W$ is set generic over $V$. If also $\lambda$-covering holds, for all sufficiently large $\lambda$, then $W$ itself is a set generic extension of $V$ (see Theorem 4.3). Example 2.7 (the example with Prikry sequences) can be extended to show that the cocovering hypothesis alone is not sufficient to get that $W$ is a set generic extension of $V$.

It is open whether the hypotheses on cardinal arithmetic in $V$ can be eliminated in 2.12 in favor of some covering hypothesis.

Question 2.13. Let $\kappa$ be regular in $V$ and assume the following hold between $V$ and $W$ :

- $\kappa$-covering for subsets of $\left(2^{\kappa^{<\kappa}}\right)^{V}$; and

- $\kappa$ and $\kappa^{+}$-cocovering.

Must every subset of $\kappa^{+}$that lies in $W$ be set generic over $V$ for a forcing of $V$ cardinality less than $\kappa$ ?

Nothing in our work to this point suggests that both $\kappa$ and $\kappa^{+}$-cocovering should be necessary for Corollary 2.12. In fact, both are necessary even to get the weaker result that every subset of $\kappa$ in $W$ is generic for a partial ordering of cardinality less than $\kappa$ in $V$. That $\kappa$-cocovering is necessary is obvious: Let $V$ be a model of GCH and let $W$ be the result of adding a Cohen subset to $\kappa$ over $V$. But what about $\kappa^{+}$? 


\subsection{SET FORCING, COVERING, AND COCOVERING}

Example 2.14. A pair of models in which the GCH holds in $V$;

- $\kappa$-cocovering holds, $\kappa$-covering holds, and $\kappa^{+}$-cocovering fails; and

- there exists a subset of $\kappa$ in $W$ that is not generic for a forcing of cardinality less than $\kappa$ in $V$.

Let $\kappa$ be ineffable in $V$. Let $\mathbb{P}=\prod_{\alpha<\kappa} \mathbb{Q}\left(\omega_{\alpha}^{+}\right)$, where $\mathbb{Q}(\nu)$ is the forcing to add a Cohen subset to $\nu$. Then $\mathbb{P}$ is a cardinal and cofinality preserving forcing over $V$ and has uniform density $\kappa^{+}$. It follows that $\kappa$-covering holds and $\kappa^{+}$-cocovering fails between $V$ and any $\mathbb{P}$-generic extension. If $G$ is $\mathbb{P}$-generic, then $a=\left\{\ll \alpha, \delta »: \exists p \in G p_{\alpha}(\delta)=1\right\}$ is a subset of $\kappa$ that is not $\mathbb{Q}$-generic, for any $\mathbb{Q} \in V$ of cardinality less than $\kappa$. All that is left is to see that $\kappa$-cocovering holds between $V$ and $V[G]$. This is where the ineffability of $\kappa$ comes in.

Work in $V$. Let $\stackrel{\circ}{f}$ be a term for a function from $\kappa$ to $\kappa$, and let $p \in \mathbb{P}$. We seek $p^{\prime} \leqslant p$ and an unbounded $S \subseteq \kappa$ such that for each $\alpha \in S$, there exists $\beta$ such that $p^{\prime} \Vdash \check{f}(\check{\alpha})=\check{\beta}$. By extending tails of $p$ in $\kappa$ many steps, if necessary, we may assume that for each $\alpha<\kappa$, then exists $\beta^{\alpha}$ and $r^{\alpha} \in \prod_{\delta \leqslant \alpha} \mathbb{Q}\left(\omega_{\delta}^{+}\right)$such that $r^{\alpha} \leqslant p \uparrow(\alpha+1)$ and $r^{\alpha} \cup p\left\lceil[\alpha+1, \kappa) \Vdash \check{f}(\check{\alpha})=\check{\beta}^{\alpha}\right.$. Code $r^{\alpha}$ as a subset $A_{\alpha}$ of $\omega_{\alpha}$. Because $\kappa$ is ineffable, there exists an unbounded $S \subseteq \kappa$ such that $A_{\alpha}=A_{\beta} \cap \omega_{\alpha}$, for all $\alpha<\beta$ in $S$. Then $r^{\beta}\left\lceil(\alpha+1)=r^{\alpha}\right.$, for $\alpha<\beta$ in $S$. So $p^{\prime}=\bigcup_{\alpha \in S} r^{\alpha}$ is as required.

Question 2.15. Assume the $G C H$ in $V$, or better yet, assume $V=L$. Does there exist a countably generated $\mathbb{P}$ such that $\mathbb{P}$ has uniform density $\omega_{2}$ and $\omega_{1}$-cocovering holds between $V$ and any $\mathbb{P}$-generic extension?

\section{3. $\pitchfork_{\lambda,<\kappa}$ and set genericity without GCH assumptions}

Let us continue to observe the notational convention that $V$ is a standard model of ZFC and that $W$ is an outer model of $V$ such that $(W ; V) \vDash$ ZFC.

It is easy to see (and was implicit in the last example of $\S 2$ ) that $\kappa$-cocovering is equivalent to the following statement: Given a function $F: \kappa \rightarrow \kappa$ that lies in $W$, there exists a function $f \in V$ such that $f \subseteq F$ and $\bigcup \operatorname{dom}(f)=\kappa$. In view of Example 2.7 (the example using Prikry sequences), one might suspect that a better form of cocovering is $\kappa$-sequence cocovering: Given a function $F: \kappa \rightarrow$ OR that lies in $W$, there exists $f \in V$ such that $f \subseteq F$ and $\bigcup \operatorname{dom}(f)=\kappa$. In addition to $\kappa$ cocovering, this principle implies a certain amount of covering. (In fact, if $\kappa$-sequence cocovering holds for all infinite $V$-regular $\kappa$, then $V=W$. But we do not need this fact in this paper.) In an effort to distinguish the roles of these characters, we chose in $\S 2$ not to work with $\kappa$-sequence cocovering. However, as Lemma 3.3 indicates, with $\pitchfork_{\lambda,<\kappa}$ we are implicitly working with some sequence cocovering.

Let $\kappa$ be regular in $V$ and let $\lambda \geqslant \kappa$. Recall from $\S 1$ that $\pitchfork_{\lambda,<\kappa}$ is the following statement:

$$
\begin{array}{ll}
\left(\pitchfork_{\lambda,<\kappa}\right) \quad \text { For each } F:[\lambda]^{<\kappa} \rightarrow \lambda \text { in } W, \\
\text { there exists } f \in V \text { such that } f \subseteq F \text { and } \bigcup \operatorname{dom}(f)=\lambda .
\end{array}
$$

Remark 3.1. Restricting to functions $F:[\lambda]^{<\kappa} \cap V \rightarrow \lambda$ results in a principle equivalent to $\pitchfork_{\lambda,<\kappa}$ because $\operatorname{dom}(f) \subseteq V$ in its conclusion. 


\section{3. $\pitchfork_{\lambda,<\kappa}$ AND SET GENERICITY WITHOUT GCH ASSUMPTIONS}

Note that, like $<\kappa$-covering and $\kappa$-cocovering, $\pitchfork_{\lambda,<\kappa}$ holds for generic extensions by forcing of size less than $\kappa$ :

Lemma 3.2. Suppose that $W=V[G]$, where $G$ is $\mathbb{P}$-generic over $V$ and $|\mathbb{P}|^{V}<\kappa$. Then $\pitchfork_{\lambda,<\kappa}$ holds, for all $\lambda \geqslant \kappa$.

Proof: Let $\stackrel{\circ}{F}$ be a term for a function from $[\lambda]^{<\kappa}$ to $\lambda$ in $V[G]$. For each $p \in \mathbb{P}$, let

$$
f_{p}=\left\{(a, \beta): a \in[\lambda]^{<\kappa} \cap V \text { and } p \Vdash \stackrel{\circ}{F}(\check{a})=\check{\beta} \text {, for some } \beta\right\} \text {. }
$$

It suffices to see that $\left\{p \in \mathbb{P}: \bigcup \operatorname{dom}\left(f_{p}\right)=\lambda\right\}$ is dense in $\mathbb{P}$. Were this set not dense, we could choose $\bar{p}$ below which it is empty. For $p \leqslant \bar{p}$, let $\gamma_{p}<\lambda$ be least such that $\gamma_{p} \notin \bigcup \operatorname{dom}\left(f_{p}\right)$. Set $a=\left\{\gamma_{p}: p \leqslant \bar{p}\right\}$. Then no condition below $\bar{p}$ forces a value for $\stackrel{\circ}{F}(\check{a})$, which is absurd.

Next, let us observe some connections between $\pitchfork_{\lambda,<\kappa}$ and cocovering.

Lemma 3.3. Assume that $\kappa$ is regular in $V$ and that $\pitchfork_{\lambda,<\kappa}$ holds.

(a) If $\kappa \leqslant \eta \leqslant \lambda$ and $\mathrm{cf}^{V}(\eta) \geqslant \kappa$, then $\eta$-cocovering holds.

(b) $\kappa$-sequence cocovering holds for $H \in W$ such that $H: \kappa \rightarrow \lambda$.

Proof: For (a), let $a$ be an unbounded subset of $\eta$ in $W$. Define $F:[\lambda]^{<\kappa} \rightarrow \lambda$ by $F(s)=\min (a \backslash \sup (s \cap \eta))$, if $\sup (s)<\eta$; let $F(s)=0$, otherwise. (We are proving $\eta$-cocovering, from which it follows that $\mathrm{cf}^{W}(\eta)=\mathrm{cf}^{V}(\eta) \geqslant \kappa$. So the "otherwise" case never actually occurs.) Let $f \in V$ be such that $f \subseteq F$ and $\bigcup \operatorname{dom}(f)=\lambda$. Then $b=\operatorname{rng}(f) \backslash\{0\}$ is an unbounded subset of $a$.

For (b), let $H: \kappa \rightarrow \lambda$ lie in $W$. Set $a=\{\ll \gamma, H(\gamma) »: \gamma<\kappa\}$. Let $\eta$ be least such that $|a \cap \eta|^{W}=\kappa$. By (a), there exists $b \in V$ such that $b$ is unbounded in $a \cap \eta$. Set $h=\{(\gamma, \delta): \ll \gamma, \delta » \in b\}$. Then $h \in V$ and $h \subseteq H$, and $\bigcup \operatorname{dom}(h)=\kappa$, since $|h|=\kappa . \square$

Lemma 3.4. Let $\kappa$ be regular in $V$. Then $\pitchfork_{\kappa,<\kappa}$ is equivalent to $\kappa$-cocovering.

Proof: We must see that $\kappa$-cocovering implies $\pitchfork_{\kappa,<\kappa}$. (The converse is handled by (a) of Lemma 3.3.) Suppose that $F:[\kappa]^{<\kappa} \rightarrow \kappa$ lies in $W$. Set $a=\{\ll \gamma, F(\gamma) »: \gamma<\kappa\}$. Let $b \in V$ be an unbounded subset of $a$ and set $f=\{(\gamma, \delta): \ll \gamma, \delta » \in b\}$. Then $f \subseteq F$ and $\bigcup \operatorname{dom}(f)=\kappa$.

The principles $\pitchfork_{\lambda,<\kappa}$ get stronger as the gap between $\kappa$ and $\lambda$ gets larger:

Lemma 3.5. Assume that $\kappa^{\prime}$ and $\kappa$ are regular in $V$, and that $\kappa^{\prime} \leqslant \kappa \leqslant \lambda \leqslant \lambda^{\prime}$. Then $\pitchfork_{\lambda^{\prime},<\kappa^{\prime}}$ implies $\pitchfork_{\lambda,<\kappa}$.

Proof: To see $\pitchfork_{\boldsymbol{\lambda},<\boldsymbol{\kappa}^{\prime}} \Rightarrow \pitchfork_{\boldsymbol{\lambda},<\boldsymbol{\kappa}}$ : Suppose that $F:[\lambda]^{<\kappa} \rightarrow \lambda$ lies in $W$. Let $F^{\prime}=$ $F \uparrow[\lambda]^{<\kappa^{\prime}}$. Let $f \in V$ be such that $f \subseteq F^{\prime}$ and $\bigcup \operatorname{dom}(f)=\lambda$. Then $f$ is as required.

To see $\pitchfork_{\boldsymbol{\lambda}^{\prime},<\kappa} \Rightarrow \pitchfork_{\boldsymbol{\lambda},<\kappa}$ : Let $F:[\lambda]^{<\kappa} \rightarrow \lambda$ lie in $W$. Define $F^{\prime}:\left[\lambda^{\prime}\right]^{<\kappa} \rightarrow \lambda^{\prime}$ by setting $F^{\prime}(a)=F(a \cap \lambda)$. Let $f^{\prime} \in V$ be such that $f^{\prime} \subseteq F^{\prime}$ and $\bigcup \operatorname{dom}\left(f^{\prime}\right)=\lambda^{\prime}$. Note that if $a, b \in \operatorname{dom}\left(f^{\prime}\right)$ and $a \cap \lambda=b \cap \lambda$, then $f^{\prime}(a)=F^{\prime}(a)=F(a \cap \lambda)=F(b \cap \lambda)=$ $F^{\prime}(b)=f^{\prime}(b)$. It follows that $f=\left\{\left(a \cap \lambda, f^{\prime}(a)\right): a \in \operatorname{dom}(f)\right\}$ is as required. 


\section{3. $\pitchfork_{\lambda,<\kappa}$ AND SET GENERICITY WITHOUT GCH ASSUMPTIONS}

The main theorem of this paper is proved in $\S 8$ :

Set Forcing Theorem. In $V$, let $\kappa, \mu$, and $\lambda$ be cardinals such that

- $\omega_{1} \leqslant \kappa<\mu \leqslant \lambda$,

- $\kappa$ is regular,

- $\operatorname{cf}(\mu)>\kappa$, and

- $\lambda^{<\mu}=\lambda$.

Assume that

- $<\kappa$-covering for subsets of $\lambda$ and

- $\pitchfork_{\lambda,<\kappa}$

hold between $V$ and $W$. Then every subset of $\mu$ in $W$ is set generic over $V$ for a forcing of $V$-cardinality less than $\kappa$.

We can now derive Corollary 2.12 from the Set Forcing Theorem.

Proof of Corollary 2.12. Let $\kappa$ be regular in $V$ and assume the following:

- $\kappa^{<\kappa}=\kappa$ and $2^{\kappa}=\kappa^{+}$in $V$ and

- both $\kappa$ and $\kappa^{+}$-cocovering hold between $V$ and $W$.

In $V$, set $\mu=\lambda=\kappa^{+}$. Then $\operatorname{cf}^{V}(\mu)>\kappa$ and $\left(\lambda^{<\mu}\right)^{V}=\lambda$. By $\kappa$-cocovering, we know that $\kappa$ is regular in $W$. Because $\lambda=\kappa^{+}, \kappa^{+}$and $\kappa$-covering for subsets of $\lambda$ is trivial. As observed in Lemma 2.9, $\kappa$-covering for subsets of $\lambda$ implies $<\kappa$-covering for subsets of $\lambda$. By Lemma 3.6 below, we also have $\pitchfork_{\kappa^{+},<\kappa}$. By the Set Forcing Theorem it follows that every subset of $\kappa^{+}$that lies on $W$ is set generic over $V$ for a forcing of $V$-cardinality less than $\kappa$.

Lemma 3.6. Let $\kappa$ be regular in $V$. Assume

- $\left(\kappa^{<\kappa}\right)^{V}=\kappa$;

- $\kappa^{+}$-covering for subsets of $\left(2^{\kappa}\right)^{V}$; and

- both $\kappa$ and $\kappa^{+}$-cocovering.

Then $\pitchfork_{\kappa^{+},<\kappa}$ holds.

Proof: In $V$, set $\mu=2^{\kappa}$ and let $\left\langle f_{\beta}: \beta<\mu\right\rangle$ enumerate all functions $f$ such that $\operatorname{dom}(f) \subseteq[\alpha]^{<\kappa}, \operatorname{rng}(f) \subseteq \alpha$, and $\bigcup \operatorname{dom}(f)=\alpha$, for some $\alpha<\kappa^{+}$. This is possible since $\kappa^{<\kappa}=\kappa$ and $2^{\kappa}=\mu$.

In $W$, fix some $F:\left[\kappa^{+}\right]^{<\kappa} \rightarrow \kappa^{+}$. Set

$$
A=\left\{\alpha<\kappa^{+}: \text {if } a \in\left([\alpha]^{<\kappa}\right)^{V} \text {, then } F(a) \in \alpha\right\} .
$$

Then $A$ is unbounded in $\kappa^{+}$by $\left(\kappa^{<\kappa}\right)^{V}=\kappa$ and that $\kappa^{+}$is regular (in $W$ by $\kappa^{+}$cocovering). If $\alpha \in A$, let $F_{\alpha}:[\alpha]^{<\kappa} \rightarrow \alpha$ by $F_{\alpha}(a)=F(a)$, if $a \in\left([\alpha]^{<\kappa}\right)^{V}$; otherwise, set $F_{\alpha}(a)=0$. From $\kappa$-cocovering, we have $\pitchfork_{\kappa,<\kappa}$. Using this, there exists $\beta(\alpha)<\mu$ such that $f_{\beta(\alpha)} \subseteq F_{\alpha}$. Set $B=\{\beta(\alpha): \alpha \in A\}$. Then $|B|^{W}=\kappa^{+}$, since $\bigcup \operatorname{dom}\left(f_{\beta(\alpha)}\right)=\alpha$, for $\alpha \in A$ (so $\beta(\alpha) \neq \beta\left(\alpha^{\prime}\right)$ when $\alpha \neq \alpha^{\prime}$ ). By $\kappa^{+}$-covering for subsets of $\mu$, there exists $C \in V$ such that $C \supseteq B$ and $|C|^{V}=\kappa^{+}$. We may assume that $C \subseteq \mu$. Let $g \in V$ be a bijection $g: C \rightarrow \kappa^{+}$. Then $g$ " $B$ is an unbounded subset of $\kappa^{+}$. Let $D \in V$ be an unbounded subset of $g$ " $B$. Set $f=\bigcup_{g(\beta) \in D} f_{\beta}$. Then $f \in V$. 


\section{3. $\pitchfork_{\lambda,<\kappa}$ AND SET GENERICITY WITHOUT GCH ASSUMPTIONS}

Also $f \subseteq F$ : If $g(\beta) \in D$, then $\beta \in B$, so $\beta=\beta(\alpha)$ where $f_{\beta(\alpha)} \subseteq F_{\alpha}$. Because $\operatorname{dom}\left(f_{\beta(\alpha)}\right) \subseteq V$, it follows that $f_{\beta} \subseteq F$.

Finally, note that $\bigcup \operatorname{dom}(f)=\kappa^{+}$: If $\gamma<\kappa^{+}$, then there exists $\alpha \in A$ such that $\alpha>\gamma$ and $g(\beta(\alpha)) \in D$, because $|D|=\kappa^{+}$. Then $f_{\beta(\alpha)} \subseteq f$, so $\gamma \in \bigcup \operatorname{dom}(f)$.

The two principal hypotheses of the Set Forcing Theorem are $\pitchfork_{\lambda,<\kappa}$ and $<\kappa$-covering for subsets of $\lambda$. Below, Lemma 3.8 shows that if $\lambda$ is not too much larger than $\kappa-$ there are no limit cardinals between $\kappa$ and $\lambda$-then the second of these hypotheses follows from the first. First, though, let us illustrate the utility of Lemma 3.8 by using it to draw the following corollary of the Set Forcing Theorem.

Corollary 3.7. In $V$, let $\kappa$ be a regular uncountable cardinal and set $\lambda=2^{\kappa}$. Assume that there are no limit cardinals (of $V$ ) between $\kappa$ and $\lambda$. If $\pitchfork_{\lambda,<\kappa}$ holds, then every subset of $\kappa^{+}$in $W$ is set generic over $V$ for a forcing of $V$-cardinality less than $\kappa$.

Proof: In the statement of the Set Forcing Theorem, set $\mu=\kappa^{+}$and $\lambda=2^{\kappa}$. The hypothesis that $<\kappa$-covering for subsets of $\lambda$ holds follows from Lemma 3.8.

Let $\boldsymbol{\kappa}^{n+}$ be the $n^{\text {th }}$ cardinal successor of $\kappa$. Using Lemma 3.3(a), note that $\pitchfork_{\kappa^{n+},<\kappa}$ implies $\eta$-cocovering, for $\kappa \leqslant \eta \leqslant \kappa^{n+}$.

Lemma 3.8. Let $\kappa$ be regular in $V$ and set $\lambda=\left(\kappa^{n+}\right)^{V}$, for some $n \in \omega$. Assume that $\eta$-cocovering holds, for $\kappa \leqslant \eta \leqslant \lambda$. Then $<\kappa$-covering for subsets of $\lambda$ holds.

Proof: The proof is essentially trivial by induction on $n$. If $n=0$, then $\lambda=\kappa$. And $<\kappa$-covering for subsets of $\kappa$ is trivial.

Let $n>0$. By the cocovering hypotheses, every $V$-cardinal from $\kappa$ through $\lambda$ is a $W$-cardinal. If $a \in W$ is a subset of $\lambda$ having $W$-cardinality less than $\kappa$. Then $\alpha=\sup (a)<\lambda$, since $\lambda$ is regular. Project $a$ to a subset of $\kappa^{(n-1)+}$ using a bijection with $\alpha$ that lies in $V$. By induction, cover the projection with a set in $V$ of cardinality less than $\kappa$. Then apply the inverse of the bijection to cover $a$.

\section{Applications}

Let us continue to observe the notational convention that $V$ is a standard model of ZFC and that $W$ is an outer model of $V$ such that $(W ; V) \vDash \mathrm{ZFC}$.

\subsection{Set generic outer models}

Our first application is to give a combinatorial characterization of those outer models $W$ such that $W=V[G]$, where $G$ is $\mathbb{P}$-generic over $V$ for some forcing $\mathbb{P} \in V$.

In the early 1970 s several such characterizations were proved (see $[\mathbf{B}]$ ). At the end of this section, we connect our Theorem 4.2 to these.

As remarked before, $\kappa$-covering is a strong property: A set of $W$-cardinality $\kappa$ is required to be covered by a set of $V$-cardinality $\kappa$. Let $\kappa$ be an infinite cardinal in $W$. A generally weaker and more familiar principle might be called

Jensen $\kappa$-covering. If $a \in W$ is a set of ordinals, then there exists $b \in V$ such that $b \supseteq a$ and $|b|^{W} \leqslant \max \left(\kappa,|a|^{W}\right)$.

It is clear that $\lambda$-covering, for all $W$-cardinals $\lambda \geqslant \kappa$, implies Jensen $\kappa$-covering. In contrast, simply collapsing $\left(\kappa^{+}\right)^{V}$ to cardinality $\kappa$ causes $\kappa$-covering to fail. However, in the presence of $\lambda$-cocovering, for all $V$-regular $\lambda \geqslant \kappa$, the two are equivalent. 


\subsection{SET GENERIC OUTER MODELS}

Remark 4.1. If every $V$-cardinal $\lambda \geqslant \kappa$ is a cardinal in $W$, then the following are equivalent:

- Jensen $\kappa$-covering

- $\lambda$-covering, for all $\lambda \geqslant \kappa$

Theorem 4.2. The following are equivalent:

(a) $W$ is a set generic extension of $V$.

(b) There exists a $W$-cardinal $\nu$ such that

- Jensen $\nu$-covering and

- $\pitchfork_{\lambda,<\nu}$, for $\lambda \geqslant \nu$,

hold between $V$ and $W$.

Proof: In light of the previous remark, $((a) \Rightarrow(b))$ follows from Lemmas 2.5 and 3.2. We must prove $((b) \Rightarrow(a))$.

Note that $W$ and $V$ have the same cardinals and cofinalities greater than or equal to $\nu$ because $\pitchfork_{\lambda,<\nu}$ implies $\eta$-cocovering, for $V$-regular $\eta$ such that $\nu \leqslant \eta \leqslant \lambda$. Thus $\lambda$ covering holds, for $\lambda \geqslant \nu$, by Remark 4.1. Let $\kappa>\nu$ be $V$-regular. Then $<\kappa$-covering follows from $\lambda$-covering, for $\nu \leqslant \lambda<\kappa$.

In $W$, let $\mu \geqslant \kappa^{<\kappa}$ be such that $\operatorname{cf}^{V}(\mu)>\kappa$. Let $\left\langle x_{\delta}: \delta<\mu\right\rangle$ enumerate $[\kappa]^{<\kappa}$, perhaps with repetitions. Let $\lambda$ be a $V$-cardinal such that $\lambda^{<\mu}=\lambda$ in $V$. Set $a=$ $\left\{\ll \delta, \xi »: \xi \in x_{\delta}\right\}$. Then $a \subseteq \mu$. By the Set Forcing Theorem, there exists $\mathbb{P} \in V$ of $V$-cardinality less than $\kappa$ and $G \in W$ such that $G$ is $\mathbb{P}$-generic over $V$ and $a \in V[G]$.

Note that $\lambda$-cocovering holds between $V[G]$ and $W$, for all $V[G]$-cardinals $\lambda: V[G]$ and $W$ have the same sets of cardinality less than $\kappa$, because $a \in V[G]$, and $\lambda$ cocovering holds between $V$ and $W$, for $\lambda \geqslant \kappa$. Similarly, $\lambda$-covering holds between $V[G]$ and $W$, for all $\lambda$. Hence $V[G]=W$ by Lemma 2.8 .

With a little assumption on the cardinal arithmetic of $V$, we get a characterization using cocovering in place of $\pitchfork_{\lambda,<\nu}$.

Theorem 4.3. Assume that in $V$ there exist unboundedly many regular $\kappa$ such that $\kappa^{<\kappa}=\kappa$ and $2^{\kappa}=\kappa^{+}$. The following are equivalent:

(a) $W$ is a set generic extension of $V$.

(b) There exists a $W$-cardinal $\nu$ such that

- Jensen $\nu$-covering and

- $\lambda$-cocovering, for $\lambda \geqslant \nu$,

hold between $V$ and $W$.

Proof: The proof is the same as for Theorem 4.2, using Corollary 2.12 in place of the Set Forcing Theorem.

We can use reflection in place of the assumption that covering and cocovering hold on a tail of cardinals. For example, let $S$ be the following $(W ; V)$-definable class:

$$
\begin{aligned}
\kappa \in S \quad \text { iff } \quad & \kappa \text { is inaccessible in } W ; \\
& \kappa \text { and } \kappa^{+} \text {-cocovering hold; and } \\
& \left(2^{\kappa}\right)^{V}=\kappa^{+} .
\end{aligned}
$$




\subsection{SET GENERIC OUTER MODELS}

Say that a class of ordinals is $\boldsymbol{\Sigma}_{\boldsymbol{n}}$-stationary if it meets every $\Sigma_{n}$-definable club class of ordinals.

Theorem 4.4. If $S$ is $\Sigma_{3}$-stationary in $(W ; V)$, then $W$ is a set generic extension of $V$.

Proof: If $S$ is $\Sigma_{3}$-stationary, then there exists $\kappa \in S$ such that $\left(W_{\kappa} ; V_{\kappa}\right) \prec_{2}(W ; V)$. Let $a \subseteq \kappa$ code $W_{\kappa}$. By Corollary 2.12, $a$ is $\mathbb{P}$-generic over $V$ for a forcing $\mathbb{P} \in V_{\kappa}$. Thus $\left(W_{\kappa} ; V_{\kappa}\right) \vDash \forall x \exists \stackrel{\circ}{x} \in V^{\mathbb{P}}\left(x=\stackrel{\circ}{x}^{G}\right)$. It follows that $W=V[G]$.

As mentioned at the beginning of this section, chacterizations of set generic outer models have been known for a long time.

Definition. The following principles are due to Vopěnka and Hájek. Let $\kappa$ be a cardinal of $V$.

$$
\begin{aligned}
\operatorname{Bd}_{V, W}(\kappa): \quad \text { If } a \in W \text { is a subset of } V & \\
& \text { then there exist } b \in W \text { and } c \in V \text { such that } \\
& a=\bigcup b \text { and } c \supseteq b \text { and }|c|^{V}<\kappa . \\
\operatorname{Apr}_{V, W}(\kappa): \quad \text { If, for some ordinal } \alpha, f: \alpha \rightarrow \text { OR lies in } W & \\
& \text { then there exists a function } g \in V \text { with domain } \alpha \text { such that } \\
& |g(\alpha)|^{V}<\kappa \text { and } f(\delta) \in g(\delta), \text { for all } \delta<\alpha .
\end{aligned}
$$

It is an easy exercise to verify that if $\kappa$ is regular in $V$, then $\operatorname{Apr}_{V, W}(\kappa)$ is equivalent to the following statement: If $\lambda \geqslant \kappa$ and $f: \lambda \rightarrow \lambda$ lies in $W$, then in $V$ there exists a club subset $K \subseteq[\lambda]^{<\kappa}$ of a such that $f$ " $a \subseteq a$.

I believe the first characterization of set generic extensions is the following theorem of Vopěnka and Hájek.

Theorem 4.5. (Vopěnka and Hájek) Let $\kappa$ be a cardinal in $V$. The following are equivalent:

- $W$ is a set generic extension of $V$ by a forcing of $V$-cardinality less than $\kappa$.

- $\operatorname{Bd}_{V, W}(\kappa)$

Soon afterwards Bukovský [B] found another, appealing characterization:

Theorem 4.6. (Bukovský) Let $\kappa$ be a cardinal in $V$. The following are equivalent:

- $W$ is a set generic extension of $V$ via a $\kappa$-c.c. forcing.

- $\operatorname{Apr}_{V, W}(\kappa)$

The proof of Bukovskýs Theorem requires that the Power Set Axiom holds in $W$. For example, if we let $W$ be the result of adding OR-many Cohen reals to $V$ (using a finite support product), then $\operatorname{Apr}_{V, W}\left(\omega_{1}\right)$ holds, even though $W$ is not a set generic extension of $V$. Consequently, we cannot expect to derive the hypotheses of our characterization, Theorem 4.2, directly from $\operatorname{Apr}_{V, W}(\kappa)$.

On the other hand, Lemma 4.7 shows that the two hypotheses of Theorem 4.2 can be derived easily from $\mathrm{Bd}_{V, W}(\kappa)$. Thus Vopěnka's Theorem can be obtained as a corollary of Theorem 4.2 . 
Lemma 4.7. Let $\kappa$ be regular in $V$. Assume $\operatorname{Bd}_{V, W}(\kappa)$. Then

(a) $\pitchfork_{\lambda,<\kappa}$ and

(b) $<\kappa$-covering for subsets of $\lambda$

hold for all $\lambda \geqslant \kappa$.

Proof: For (a), let $F:[\lambda]^{<\kappa} \rightarrow \lambda$ lie in $W$. Let $b \in W$ and $c \in V$ be such that $F \uparrow\left([\lambda]^{<\kappa}\right)^{V}=\bigcup b$ and $c \supseteq b$ and $|c|^{V}<\kappa$. It suffices to see that there exists $f \in b$ such that $\bigcup \operatorname{dom}(f)=\lambda$. Suppose not. In $V$, let $c^{\prime}=\{f \in c: \bigcup \operatorname{dom}(f) \neq \lambda\}$. Then $b \subseteq c^{\prime}$. For $f \in c^{\prime}$, let $\gamma_{f}$ be least in $\lambda \backslash \bigcup \operatorname{dom}(f)$. Let $s=\left\{\gamma_{f}: f \in c^{\prime}\right\}$. Then $s \in[\lambda]^{<\kappa}$, but $s \notin \operatorname{dom}(F)$, which is absurd.

For (b), proceed by induction on $\lambda$. Let $A \in W$ be a subset of $\lambda$ such that $|A|^{W}<\kappa$. We may assume that $A$ is unbounded in $\lambda$ and that $\lambda$ is a cardinal in $V$. Let $\left\langle\alpha_{\delta}: \delta\langle\eta\rangle \in W\right.$ be increasing and cofinal in $\lambda$. Then $\eta<\kappa$. We may assume that some element of $A$ lies between $\alpha_{\delta}$ and $\alpha_{\delta+1}$, for each $\delta<\eta$.

By induction, choose $B_{\delta} \in V$ such that $B_{\delta} \supseteq A \cap \alpha_{\delta}$ and $\left|B_{\delta}\right|^{V}<\kappa$. We may assume that $B_{\delta} \subseteq \alpha_{\delta}$. Set $a=\left\{B_{\delta}: \delta<\eta\right\}$. Using $\operatorname{Bd}_{V, W}(\kappa)$, let $b \in W$ and $c \in V$ be such that $a=\bigcup b$ and $c \supseteq b$ and $|c|^{V}<\kappa$. Note that if $x \in b$, then $x \subseteq a$, so ot $\{\sup (B): B \in x\} \leqslant \eta$. By our choice of $\left\langle\alpha_{\delta}: \delta<\eta\right\rangle$ it follows that $|x|^{V} \leqslant \eta<\kappa$.

There are two cases to consider. First, suppose that there exists $x \in b$ such that $|x|^{V}=\eta$. Then $\bigcup x \supseteq A$. Since $|x|^{V}<\kappa$ and $\left|B_{\delta}\right|^{V}<\kappa$, for each $B_{\delta} \in x$, it follows that $|\bigcup x|^{V}<\kappa$, as required for $<\kappa$-covering.

In the second case, suppose that every $x \in b$ has $V$-cardinality less than $\kappa$. By thinning $c$, if necessary, we may assume that every $x \in c$ has the same property. Similarly, if $B \in x \in b$, then $|B|^{V}<\kappa$, so we may assume that this holds for all $B \in x \in c$, as well. Then $A \subseteq \bigcup a \subseteq \bigcup \bigcup b \subseteq \bigcup \bigcup c$. And $\bigcup \bigcup c=\bigcup_{x \in c} \bigcup_{B \in x} B$ is, in $V$, a union of fewer than $\kappa$ sets, each of cardinality less than $\kappa$.

\subsection{Supercompact cardinals}

In deference to standard notation, let us write " $\mathcal{P}_{\kappa} \lambda$ " for $[\lambda]^{<\kappa}$ in this section.

Lemma 4.8. Working in $(W ; V)$, suppose that

- $D$ is a fine filter on $\mathcal{P}_{\kappa} \lambda$, that

- $\left(\mathcal{P}_{\kappa} \lambda\right)^{V} \in D$, and that,

- for each $f: \mathcal{P}_{\kappa} \lambda \rightarrow \lambda$, there exists $A \in D$ such that $f\lceil A \in V$.

Then $\left(\mathcal{P}_{\kappa} \lambda\right)^{V}=\mathcal{P}_{\kappa} \lambda \cap V$ and both $\pitchfork_{\lambda,<\kappa}$ and $<\kappa$-covering for subsets of $\lambda$ hold between $V$ and $W$.

Proof: Let us begin by checking $<\kappa$-covering for subsets of $\lambda$. Suppose that $a \in$ $\mathcal{P}_{\kappa} \lambda$. Then $\hat{a}=\left\{b \in \mathcal{P}_{\kappa} \lambda: b \supseteq a\right\} \in D$, since $D$ is fine. Consequently, there exists $b \in \hat{a} \cap\left(\mathcal{P}_{\kappa} \lambda\right)^{V}$, as required for $<\kappa$-covering.

From $<\kappa$-covering for subsets of $\lambda$, it follows that $\left(\mathcal{P}_{\kappa} \lambda\right)^{V}=\mathcal{P}_{\kappa} \lambda \cap V$.

Finally, we must check $\pitchfork_{\lambda,<\kappa}$. Let $F: \mathcal{P}_{\kappa} \lambda \rightarrow \lambda$ lie in $W$. Let $A \in D$ be such that $f=F\lceil A \in V$. If $\gamma<\lambda$, then $\widehat{\{\gamma\}} \in D$, so $A \cap \widehat{\{\gamma\}} \neq \emptyset$. Because $\gamma<\lambda$ was arbitrary, it follows that $\bigcup \operatorname{dom}(f)=\lambda$. 


\subsection{SUPERCOMPACT CARDINALS}

If $D$ is a $\kappa$-complete ultrafilter on $\mathcal{P}_{\kappa} \lambda$, let $j_{D}$ be the associated ultrapower embedding.

Definition. Assume that $D \in V$ is a normal fine $\kappa$-complete ultrafilter on $\left(\mathcal{P}_{\kappa} \lambda\right)^{V}$. In $(W ; V)$ define

$$
\widehat{D}=\left\{A \subseteq \mathcal{P}_{\kappa} \lambda: \exists B \in D B \subseteq A\right\} .
$$

Recall from $\S 1$ that $D$ automatically extends in $W$ if,

- in $W, \widehat{D}$ is a normal fine $\kappa$-complete ultrafilter on $\mathcal{P}_{\kappa} \lambda$ and

- $j_{\widehat{D}} \uparrow V=j_{D}$.

Remark 4.9. If $W=V[G]$, where $G$ is $\mathbb{P}$-generic over $V$, for some partial ordering $\mathbb{P} \in V_{\kappa}$, then any normal fine $\kappa$-complete ultrafilter $D \in V$ on $\left(\mathcal{P}_{\kappa} \lambda\right)^{V}$ automatically extends. Theorems 4.10 and 4.11 provide a converse to this observation.

Theorem 4.10. In $V$, let $\lambda \geqslant \mu>\kappa$ be uncountable cardinals such that $\kappa$ is regular, $\operatorname{cf}(\mu)>\kappa$, and $\lambda^{<\mu}=\lambda$. If there exists a normal fine $\kappa$-complete ultrafilter $D \in V$ on $\left(\mathcal{P}_{\kappa} \lambda\right)^{V}$ that automatically extends in $W$, then each subset of $\mu$ that lies in $W$ is $\mathbb{P}$-generic over $V$, for some forcing $\mathbb{P} \in V_{\kappa}$.

Proof: The plan is to verify the hypotheses of Lemma 4.8 in order to get $<\kappa$-covering for subsets of $\lambda$ and $\pitchfork_{\lambda,<\kappa}$. Then Theorem 4.10 follows from the Set Forcing Theorem.

By the definition of $\widehat{D}$, we know that $\left(\mathcal{P}_{\kappa} \lambda\right)^{V} \in \widehat{D}$.

Factor $j_{D}$ and $j_{\widehat{D}}$ as $j_{D}=k \circ e$ and $j_{\widehat{D}}=\hat{k} \circ \hat{e}$, where

$$
V \stackrel{e}{\longrightarrow} \operatorname{Ult}_{D}(V) \stackrel{k}{\longrightarrow} N \quad \text { and } \quad W \stackrel{\hat{e}}{\longrightarrow} \mathrm{Ult}_{\widehat{D}}(W) \stackrel{\hat{k}}{\longrightarrow} M
$$

and $e$ and $\hat{e}$ are canonical embeddings and $k$ and $\hat{k}$ are transitive collapses.

Work in $(W ; V)$. Let $f: \mathcal{P}_{\kappa} \lambda \rightarrow \lambda$. Then $\hat{k}\left([f]_{\widehat{D}}\right) \in j_{\widehat{D}}(\lambda)$. Because $k$ is onto $\mathrm{OR}^{V}=\mathrm{OR}^{W}$, there exists $[g]_{D} \in \operatorname{Ult}_{D}(V)$ such that $k\left([g]_{D}\right)=\hat{k}\left([f]_{\widehat{D}}\right)$. Because $j_{D}(\lambda)=j_{\widehat{D}}(\lambda)$, we may assume that $g:\left(\mathcal{P}_{\kappa} \lambda\right)^{V} \rightarrow \lambda$. Because $D$ and $\widehat{D}$ are normal,

$$
\begin{aligned}
& k\left([g]_{D}\right)=j_{D}(g)\left(j_{D} " \lambda\right) \text { and } \\
& \hat{k}\left([f]_{\widehat{D}}\right)=j_{\widehat{D}}(f)\left(j_{\widehat{D}} " \lambda\right) .
\end{aligned}
$$

Thus $j_{\widehat{D}} " \lambda=j_{D} " \lambda \in j_{\widehat{D}}\left(\left\{a \in\left(\mathcal{P}_{\kappa} \lambda\right)^{V}: g(a)=f(a)\right\}\right)$. Consequently, there exists $A \in \widehat{D}$ such that $f \uparrow A=g \uparrow A$. Let $B \in D$ be such that $B \subseteq A$. Then $f \uparrow B=g \uparrow B \in V$.

We conclude that $<\kappa$-covering for subsets of $\lambda$ and $\pitchfork_{\lambda,<\kappa}$ hold, using Lemma 4.8. The Set Forcing Theorem then provides the desired conclusion.

Theorem 4.11. Suppose that for every $\lambda \geqslant \kappa$ there exists a normal fine $\kappa$-complete ultrafilter $D \in V$ on $\left(\mathcal{P}_{\kappa} \lambda\right)^{V}$ that automatically extends in $W$. Then there exists a forcing $\mathbb{P} \in V_{\kappa}$ and a filter $G$ such that $G$ is $\mathbb{P}$-generic over $V$ and $W=V[G]$.

Proof: Note that $\left|W_{\kappa}\right|^{W}=\kappa$, since $\kappa$ is inaccessible in $W$. Let $a \in W$ be a subset of $\kappa$ coding $W_{\kappa}$. By Theorem 4.10, there exists $\mathbb{P} \in V_{\kappa}$ and a $G \in W$ such that $G$ is $\mathbb{P}$-generic over $V$ and $a \in V[G]$. Thus

$$
\left(W_{\kappa} ; V_{\kappa}\right) \vDash \forall x \exists \grave{x} \in V^{\mathbb{P}}\left(x=\grave{x}^{G}\right) .
$$


It suffices to see that $\left(W_{\kappa} ; V_{\kappa}\right) \prec_{2}(W ; V)$. This follows from the observation that, given any $b \in W$, there exists $j_{\widehat{D}}:(W ; V) \rightarrow(M ; N)$ with critical point $\kappa$ and $b \in\left(W_{j_{\widehat{D}}(\kappa)}\right)^{M}$ and $N \subseteq V$. (Choose $\lambda$ sufficiently large and let $\widehat{D}$ be the automatic extension of a normal fine $\kappa$-complete ultrafilter $D \in V$ on $\left(\mathcal{P}_{\kappa} \lambda\right)^{V}$. Then $N \subseteq V$ because $j_{\widehat{D}} \uparrow V=j_{D}$ and $j_{D}: V \rightarrow N \subseteq V$.)

Remark 4.12. The analog of Theorem 4.10 for measures on $\kappa$ is false. More precisely, assume that $D \in V$ is a normal $\kappa$-complete ultrafilter on $\kappa$. In $(W ; V)$ define

$$
\widehat{D}=\{A \subseteq \kappa: \exists B \in D B \subseteq A\} .
$$

Say that D automatically extends in $W$ if,

- in $W, \widehat{D}$ is a normal $\kappa$-complete ultrafilter and

- $j_{\widehat{D}} \uparrow V=j_{D}$.

Again, if $W=V[G]$, where $G$ is $\mathbb{P}$-generic over $V$ for some forcing $\mathbb{P} \in V_{\kappa}$, then any normal $\kappa$-complete ultrafilter on $\kappa$ in $V$ automatically extends in $W$. However, we have this

Proposition 4.13. In $V$, assume the $G C H$ and let $D$ be a normal $\kappa$-complete ultrafilter on $\kappa$. There exists a set generic extension $V[G]$ of $V$ (via a forcing of $V$-cardinality $\kappa^{+}$) such that

- $D$ automatically extends in $V[G]$, but

- there exists a subset of $\kappa$ in $V[G]$ that is not generic for a forcing of cardinality less than $\kappa$ in $V$.

Proof: In $V$, let $D$ be a normal $\kappa$-complete ultrafilter on $\kappa$. Let $\mathbb{P}$ be the forcing of Example 2.14. That is, $\mathbb{P}=\prod_{\alpha<\kappa} \mathbb{Q}\left(\omega_{\alpha}^{+}\right)$, where $\mathbb{Q}(\nu)$ is the forcing to add a Cohen subset to $\nu$.

Let $G$ be $\mathbb{P}$-generic over $V$. By the arguments of Example 2.14, we have

(1) there exists a subset of $\kappa$ in $V[G]$ that is not generic for a forcing of cardinality less than $\kappa$ in $V$, and

(2) if $f: \kappa \rightarrow V$ lies in $V[G]$, then there exists $A \in D$ such that $f\lceil A \in V$.

Let us use (2) to show that $D$ automatically extends in $V[G]$.

To see that $\widehat{D}$ is an ultrafilter, let $A \in V[G]$ be a subset of $\kappa$. Let $f: \kappa \rightarrow 2$ be its characteristic function. Let $B \in D$ be such that $f\lceil B \in V$. Because $D$ is normal in $V$, there exists $C \in D$ such that $C \subseteq B$ and $f$ is constant on $C$. Then either $C \subseteq A$ or $C \subseteq(\kappa \backslash A)$.

Similarly, it is easy to see that $\widehat{D}$ is normal and $\kappa$-complete.

Finally, we must check that $j_{\widehat{D}}\left\lceil V=j_{D}\right.$. For this, it suffices to see that

$$
\mathrm{Ult}_{D}(V)^{V} \cong \operatorname{Ult}_{\widehat{D}}(V)^{V[G]} .
$$

Define $F: \operatorname{Ult}_{D}(V)^{V} \rightarrow \operatorname{Ult}_{\widehat{D}}(V)^{V[G]}$ by

$$
F\left([f]_{D}\right)=[f]_{\widehat{D}}
$$




\subsection{SUPERCOMPACT CARDINALS}

for $f: \kappa \rightarrow V$ that lie in $V$. Note that $F$ is well-defined because $D \subseteq \widehat{D}$. Similarly, note that $F$ is an isomorphic embedding. Finally, observe that $F$ is onto: If $f: \kappa \rightarrow V$ lies in $V[G]$, then there exists $A \in D$ such that $f \nmid A \in V$. Let $g \in V$ be such that $g: \kappa \rightarrow V$ and $g \supseteq f\left\lceil A\right.$. Then $F\left([g]_{D}\right)=[g]_{\widehat{D}}=[f]_{\widehat{D}}$.

\section{Superdensity reduction}

In this and subsequent sections, we work under the following

\section{Assumptions.}

- $\mathbb{P}$ is either an element of $V$ or a $V$-definable partial ordering. Assume, without loss of generality, that $\mathbb{P}$ is separative and is closed under finite meets. That is, any finite subset of $\mathbb{P}$ has a greatest lower bound,

- $\mathbb{Q} \subseteq \mathbb{P}$ is, in $V$, a set-complete Boolean algebra with $\mathbb{0}$ removed, and $\mathbb{Q}$ is complete in $\mathbb{P}$. That is, if $q \in \mathbb{Q}$, then there exists an element $-q \in \mathbb{Q}$ such that, for all $p \in \mathbb{P}, p$ is incompatible with $q$ iff $p \leqslant-q$. Also, if $d \subseteq \mathbb{Q}$ is a set, then there exists a condition $\bigvee d \in \mathbb{Q}$ such that, for all $p \in \mathbb{P}, p \leqslant \bigvee d$ iff $d$ is predense with respect to $p$. The subordering $\mathbb{Q}$ is also perhaps a proper class.

- $G$ is a filter on $\mathbb{P}$ that is internally generic over $V$. That is,

- if $p \in G$ and $\bar{p} \geqslant p$, then $\bar{p} \in G$, and

- if $p$ and $p^{\prime}$ lie in $G$, then there exists $p^{\prime \prime} \in G$ extending both $p$ and $p^{\prime}$, and $G$ is internally generic, that is,

- if $d \in V$ is a set of conditions in $\mathbb{P}$, then either $G \cap d \neq \emptyset$ or there exists $p \in G$ that is incompatible with every element of $d$.

As far as application in this paper go, we might as well assume that $\mathbb{P}=\mathbb{Q}$ and dispense with one or the other name. In $[\mathbf{S}]$ it is shown that every "sufficiently nonminimal" outer model of $V$ is an extension that is generic with respect to the language of set theory. The forcing that witnesses this has the structure described in our Assumptions. Another application of the main theorem of this paper is to factor that forcing.

In this section,

- suppose that $\mathbb{R} \subseteq \mathbb{Q}$ is a set and $\mathbb{R}$ is closed under $\Lambda$. That is, if $x \subseteq \mathbb{R}$ and there exists $p \leqslant q$, for all $q \in x$, then $\bigwedge x \in \mathbb{R}$.

Define $h_{\mathbb{R}}: \mathbb{P} \rightarrow \mathbb{R}$ by

$$
h_{\mathbb{R}}(p)=\bigwedge\{r \in \mathbb{R}: p \leqslant r\} .
$$

Say that $e \subseteq \mathbb{R}$ is superdense in $\mathbb{R}$ with respect to $\bar{p} \in \mathbb{P}$ if, given any $p \leqslant \bar{p}$ in $\mathbb{P}$, there exists $q \in e$ such that $q \sim p$ and $q \leqslant h_{\mathbb{R}}(p)$.

Note that if $e$ is superdense with respect to $\bar{p}$, then $e$ is predense with respect to $\bar{p}$, that is $\bar{p} \leqslant \bigvee e$.

Let

$$
\Pi(\mathbb{R})=\{\bigwedge x: x \subseteq \mathbb{R} \cup\{-r: r \in \mathbb{R}\}\} .
$$

Then $\mathbb{R} \subseteq \Pi(\mathbb{R})$, since $\bigwedge\{r\}=r$, and $\Pi(\mathbb{R})$ is closed under $\bigwedge$. Let $\Pi^{2}(\mathbb{R})=\Pi(\Pi(\mathbb{R}))$. 


\section{SUPERDENSITY REDUCTION}

Granted that $\mathbb{R}$ is a set, so are $\Pi(\mathbb{R})$ and $\Pi^{2}(\mathbb{R})$, and so forth. Iterating the $\Pi$ operation and taking unions at limits generates the smallest set-complete Boolean algebra that is complete in $\mathbb{P}$ and extends $\mathbb{R}$. At successor stages, the ordering is closed under $\Lambda$; at limits, under complementation. In general, the process iterates through the ordinals without closing off. The plan for our proof is to use $\pitchfork_{\lambda,<\kappa}$ and $<\kappa$-covering for subsets of $\lambda$ to find fixed points in this process.

Recall the principle $\pitchfork_{\lambda,<\kappa}$ : If $W$ is an outer model of $V$ and $F:[\lambda]^{<\kappa} \rightarrow \lambda$ lies in $W$, then there exists $f \in V$ such that $f \subseteq F$ and $\bigcup \operatorname{dom}(f)=\lambda$.

Superdensity Reduction - Lemma 5.1. In $V$, let $\kappa \geqslant \omega_{1}$ be a regular cardinal and let $\lambda \geqslant \kappa$ be a cardinal. Assume that $\pitchfork_{\lambda,<\kappa}$ holds between $V$ and $V[G]$. Suppose that $e \in V$ is a subset of $\mathbb{R}$ such that $|e|^{V} \leqslant \lambda$ and $e$ is superdense in $\mathbb{R}$ with respect to $\tilde{p} \in G \cap \Pi(\mathbb{R})$. Then there exists $d \in[e]^{<\kappa}$ and $p^{*} \in G \cap \Pi^{2}(\mathbb{R})$ such that $d$ is superdense in $\mathbb{R}$ with respect to $p^{*}$.

Proof: Let $\kappa, \lambda, G, e$, and $\tilde{p}$ be as in the statement of the lemma. Note first that it suffices to prove the lemma without the requirement that $p^{*} \in \Pi^{2}(\mathbb{R})$ :

Claim 5.2. It suffices to find $d \in[e]^{<\kappa}$ and $p^{*} \in G$ such that $d$ is superdense in $\mathbb{R}$ with respect to $p^{*}$.

Proof of the Claim: Set $p=h_{\Pi^{2}(\mathbb{R})}\left(p^{*}\right)$. Then $p \in G \cap \Pi^{2}(\mathbb{R})$. We must see that $d$ is superdense in $\mathbb{R}$ with respect to $p$. Let $p^{\prime} \leqslant p$. Then $p^{*} \sim h_{\Pi(\mathbb{R})}\left(p^{\prime}\right)$, since otherwise $p^{*} \leqslant-h_{\Pi(\mathbb{R})}\left(p^{\prime}\right) \in \Pi^{2}(\mathbb{R})$, and then $p \leqslant-h_{\Pi(\mathbb{R})}\left(p^{\prime}\right)$, contradicting that $p^{\prime} \leqslant p$. Let $p^{\prime \prime}$ extend both $p^{*}$ and $h_{\Pi(\mathbb{R})}\left(p^{\prime}\right)$. Because $d$ is superdense in $\mathbb{R}$ with respect to $p^{*}$, there exists $q \in d$ such that $q \sim p^{\prime \prime}$ and $q \leqslant h_{\mathbb{R}}\left(p^{\prime \prime}\right)$. Now $h_{\mathbb{R}}\left(p^{\prime}\right) \geqslant h_{\mathbb{R}}\left(p^{\prime \prime}\right)$ because $h_{\Pi(\mathbb{R})}\left(p^{\prime}\right) \geqslant p^{\prime \prime}$. Hence $q \leqslant h_{\mathbb{R}}\left(p^{\prime}\right)$. And $q \sim p^{\prime}$, since otherwise $p^{\prime} \leqslant-q$. In this case $h_{\Pi(\mathbb{R})}\left(p^{\prime}\right) \leqslant-q$. But $h_{\Pi(\mathbb{R})}\left(p^{\prime}\right) \sim q$ since $p^{\prime \prime} \leqslant h_{\Pi(\mathbb{R})}\left(p^{\prime}\right)$ and $p^{\prime \prime} \sim q$.

In $V$, let

$$
\left\langle q_{\alpha}: \alpha<\lambda\right\rangle
$$

be an enumeration of $e$.

For a contradiction (to $\pitchfork_{\lambda,<\kappa}$ ), assume that for all $d \in[e]^{<\kappa}$ and all $p^{*} \in G, d$ is not superdense in $\mathbb{R}$ with respect to $p^{*}$. We have that

$$
\left\{\begin{array}{l}
\text { if } p^{*} \in G \text { and } d \in[e]^{<\kappa} \\
\text { then there exists } p \leqslant p^{*}
\end{array}\right.
$$

The plan is to find a "simple" term $\stackrel{\circ}{F}$ for the function $F:\left([\lambda]^{<\kappa}\right)^{V} \rightarrow \lambda$ defined in $V[G]$ by

$$
F(s)=\text { the least } \beta \text { such that } q_{\beta} \in G \text { and } \forall \gamma \in s\left(q_{\gamma} \in G \rightarrow q_{\gamma} \nless q_{\beta}\right)
$$

and argue from $(\dagger)$ that there is no $f \in V$ such that $f \subseteq F$ and $\bigcup \operatorname{dom}(f)=\lambda$. This contradicts $\pitchfork_{\lambda,<\kappa}$. We must be careful carrying out this plan because we are assuming only that $G$ is internally generic.

For $s \in[\lambda]^{<\kappa}$, set

$$
B_{s}=\left\{q \in \Pi(\mathbb{R}): \exists \beta\left(q \leqslant q_{\beta} \wedge \forall \gamma \in s\left(q \sim q_{\gamma} \rightarrow q_{\gamma} \nless q_{\beta}\right)\right)\right\} .
$$




\section{SUPERDENSITY REDUCTION}

Claim 5.3. $B_{s} \cap G \neq \emptyset$

Proof: For a contradiction, suppose that $G \cap B_{s}=\emptyset$. Then $\bigwedge_{q \in B_{s}}-q \in G$, since otherwise $\bigvee B_{s} \in G$. In this case, the set of conditions $B_{s}$ would be predense with respect to this condition in $G$, and we would have $B_{s} \cap G \neq \emptyset$.

Let $p^{*} \in G$ extend both $\tilde{p}$ and $\bigwedge_{q \in B_{s}}-q$. By $(\dagger)$ there exists $p^{\prime} \leqslant p^{*}$ such that

$$
\forall \gamma \in s\left(p^{\prime} \sim q_{\gamma} \rightarrow q_{\gamma} \nless h_{\mathbb{R}}\left(p^{\prime}\right)\right) .
$$

Now $p^{\prime} \leqslant p^{*} \leqslant \tilde{p}$, hence by the superdensity of $e$, there exists $q_{\beta}$ such that $p^{\prime} \sim q_{\beta}$ and $q_{\beta} \leqslant h_{\mathbb{R}}\left(p^{\prime}\right)$. Let $p$ extend both $p^{\prime}$ and $q_{\beta}$. Set $q=h_{\Pi(\mathbb{R})}(p)$. Then $q \leqslant q_{\beta}$, since $p \leqslant q_{\beta} \in \mathbb{R}$ and $q=h_{\Pi(\mathbb{R})}(p)$.

Fix $\gamma \in s$ and suppose that $q_{\gamma} \leqslant q_{\beta}$. We maintain that $q \mid q_{\gamma}$. This will show that $q \in B_{s}$, which is impossible since $p \leqslant q$ and $p \leqslant \bigwedge_{q \in B_{s}}-q$. Now $q_{\gamma} \leqslant q_{\beta} \leqslant h_{\mathbb{R}}\left(p^{\prime}\right)$. Thus $p^{\prime} \mid q_{\gamma}$ by $(*)$. So $p \leqslant-q_{\gamma}$ since $p \leqslant p^{\prime}$. But $-q_{\gamma} \in \Pi(\mathbb{R})$, so $q \leqslant-q_{\gamma}$ since $q=h_{\Pi(\mathbb{R})}(p)$. Thus $q \mid q_{\gamma}$.

If $q \in B_{s}$, let $\beta(q)$ be the least $\beta$ witnessing that $q \in B_{s}$. Then for $q \in B_{s}$, we have the following:

(1) $q \leqslant q_{\beta(q)}$;

(2) $\forall \gamma \in s\left(q \sim q_{\gamma} \rightarrow q_{\gamma} \nless q_{\beta(q)}\right)$; and

(3) $\forall \beta^{\prime}<\beta(q)\left(q \leqslant q_{\beta^{\prime}} \rightarrow \exists \gamma \in s\left(q \sim q_{\gamma} \wedge q_{\gamma} \leqslant q_{\beta^{\prime}}\right)\right)$.

Claim 5.4. Suppose that $q \in B_{s}$ and $q^{\prime} \leqslant q$, where $q^{\prime} \in \Pi(\mathbb{R})$. Then $q^{\prime} \in B_{s}$ and $\beta\left(q^{\prime}\right) \leqslant \beta(q)$.

Proof: Set $\beta=\beta(q)$. Then $q^{\prime} \leqslant q \leqslant q_{\beta}$ and $\forall \gamma \in s\left(q \sim q_{\gamma} \rightarrow q_{\gamma} \nless q_{\beta}\right)$. It follows that $\beta$ witnesses that $q^{\prime} \in B_{s}$.

In $V$, for $s \in[\lambda]^{<\kappa}$ and $\beta<\lambda$, set

$$
p_{s \beta}=\bigvee\left\{q: q \in B_{s} \text { and } \beta(q)=\beta\right\} \wedge \bigwedge\left\{-q: q \in B_{s} \text { and } \beta(q)<\beta\right\},
$$

if this set Boolean combination is non- $\mathbb{0}$. Otherwise $p_{s \beta}$ is undefined.

Note that if $\beta \neq \beta^{\prime}$ and both $p_{s \beta}$ and $p_{s \beta^{\prime}}$ are defined, then $p_{s \beta} \mid p_{s \beta^{\prime}}$.

Set

$$
\stackrel{\circ}{F}=\left\{\left((s, \beta)^{\swarrow}, p_{s \beta}\right): s \in[\lambda]^{<\kappa} \text { and } \beta<\lambda \text { and } p_{s \beta} \text { is defined }\right\} .
$$

Then $\stackrel{\circ}{F}$ is a Shoenfield term in $V^{\mathbb{Q}}$.

Claim 5.5. $\stackrel{\circ}{F}^{G}:\left([\lambda]^{<\kappa}\right)^{V} \rightarrow \lambda$.

Proof: Fix $s \in\left([\lambda]^{<\kappa}\right)^{V}$. Since $p_{s \beta} \mid p_{s \beta^{\prime}}$ when $\beta \neq \beta^{\prime}$ (and both $p_{s \beta}$ and $p_{s \beta^{\prime}}$ are defined), there exists at most one $\beta$ such that $p_{s \beta} \in G$. It follows that $\stackrel{\circ}{F}^{G}$ is a function.

Let $\beta$ be least such that $\beta=\beta(q)$, for some $q \in B_{s} \cap G$. Then $p_{s \beta} \in G$. Hence $(s, \beta) \in \stackrel{\circ}{F}^{G}$. 


\section{SUPERDENSITY REDUCTION}

Because we assume only that $G$ is an internally generic filter on $\mathbb{P}$, many statements may hold in $V[G]$ that are not forced by any condition in $G$. On the other hand, $\stackrel{\circ}{F}$ is a term of a special form: Say that a Shoenfield term $\stackrel{z}{z}$ of the forcing language for $\mathbb{P}$ is a simple $\mathbb{Q}$-term for a subset of $a \in V$ if there exist sets of conditions $F_{c} \subseteq \mathbb{Q}$, for $c \in a$, such that

$$
\stackrel{\check{z}}{=}=\left\{(\check{c}, q): c \in a \text { and } q \in F_{c}\right\} .
$$

Claim 5.6. Assume that $\stackrel{2}{z}$ is a simple $\mathbb{Q}$-term for a subset of $a \in V$ and that $b \subseteq \dot{z}^{G}$, where $b \in V$. There exists $p \in G$ such that $F_{c}$ is predense with respect to $p$, for all $c \in b$.

Proof: Suppose not. Then $\left\{-\bigvee F_{c}: c \in b\right.$ and $\left.\bigvee F_{c} \neq \mathbb{1}\right\}$ is predense with respect to $p$, for all $p \in G$. Because $G$ is internally generic, $-\bigvee F_{c} \in G$, for some $c \in b$. But then $c \notin \stackrel{i}{z}^{G}$.

The following claim contradicts that $\pitchfork_{\lambda,<\kappa}$ holds between $V$ and $V[G]$, completing the proof of the Superdensity Reduction Lemma.

Claim 5.7. If $f \in V$ and $f \subseteq \stackrel{\circ}{F}^{G}$, then there exists $\gamma<\lambda$ such that $\gamma \notin \bigcup \operatorname{dom}(f)$.

Proof: By the previous claim, there exists $p \in G$ such that $F_{(s, f(s))}=\left\{p_{s f(s)}\right\}$ is predense with respect to $p$, for all $s \in \operatorname{dom}(f)$. Thus $p \leqslant p_{s f(s)}$, for all $s \in \operatorname{dom}(f)$. Now $p_{s f(s)} \leqslant \bigvee\left\{q \in B_{s}: \beta(q)=f(s)\right\}$, and each condition in this set extends $q_{f(s)}$ by the definition of $\beta(q)$. It follows that $p \leqslant q_{f(s)}$.

We may assume that $p \leqslant \tilde{p}$, since $G$ is a filter. Using that $e$ is superdense in $\mathbb{R}$ with respect to $\tilde{p}$, let $\gamma$ be such that $q_{\gamma} \sim p$ and $q_{\gamma} \leqslant h_{\mathbb{R}}(p)$. We claim that $\gamma \notin \bigcup \operatorname{dom}(f)$.

Fix $s \in \operatorname{dom}(f)$. Let $p^{\prime}$ extend both $p$ and $q_{\gamma}$. Let $q \in B_{s}$ be such that $\beta(q)=f(s)$ and $q \sim p^{\prime}$. (Recall that $p \leqslant \bigvee\left\{q \in B_{s}: \beta(q)=f(s)\right\}$.) Then $q \sim q_{\gamma}$ because $q \sim p^{\prime} \leqslant q_{\gamma}$. And $q_{\gamma} \leqslant q_{f(s)}$ because $q_{\gamma} \leqslant h_{\mathbb{R}}(p)$ and $p \leqslant q_{f(s)}$. By the definition of $\beta(q)=f(s)$, we know that $\forall \gamma \in s\left(q \sim q_{\gamma} \rightarrow q_{\gamma} \nless q_{f(s)}\right)$. It follows that $\gamma \notin s$. Since $s \in \operatorname{dom}(f)$ was arbitrary, we conclude $\gamma \notin \bigcup \operatorname{dom}(f)$, as required to finish the proof of the Claim and, with it, the proof of the Superdensity Reduction Lemma.

\section{Iterated reduction}

In this section, we continue to work under the following

\section{Assumptions.}

- $\mathbb{P}$ is either an element of $V$ or a $V$-definable partial ordering that is separative and is closed under finite meets.

- $\mathbb{Q} \subseteq \mathbb{P}$ is, in $V$, a set-complete Boolean algebra with $\mathbb{O}$ removed, and $\mathbb{Q}$ is complete in $\mathbb{P}$.

- $G$ is a filter on $\mathbb{P}$ that is internally generic over $V$.

Recall that if $W$ is an outer model of $V$ and $\kappa<\lambda$ are cardinals of $V$, then $<\kappa$ covering for subsets of $\lambda$ holds provided that, given $x \subseteq \lambda$ in $W$ with $|x|^{W}<\kappa$, there exists $y \in V$ such that $y \supseteq x$ and $|y|^{V}<\kappa$. 


\section{ITERATED REDUCTION}

Iterated Superdensity Reduction - Lemma 6.1. In $V$, let $\kappa \geqslant \omega_{1}$ be regular, let $\lambda \geqslant \mu>\kappa$ be cardinals such that $\lambda^{<\mu}=\lambda$. Let $\left\langle q_{\alpha}: \alpha<\mu\right\rangle$ be a sequence of conditions in $\mathbb{Q}$. For $\alpha<\mu$, there exists $E^{\alpha} \subseteq \mathbb{Q}$ and $e_{p}^{\alpha} \subseteq \mathbb{Q}$, for $p \in E^{\alpha}$, satisfying the following:

- $E^{\alpha}$ is an antichain (perhaps not maximal) and $\left|E^{\alpha}\right| \leqslant \lambda$.

- Each $p \in E^{\alpha+1}$ meets $E^{\alpha}$. More generally, let $E^{\beta}\left\lceil p=\left\{q \in E^{\beta}: q \sim p\right\}\right.$. If $p \in E^{\alpha}$, then $\mid E^{\beta}\lceil p \mid<\kappa$, for $\beta<\alpha$.

- $e_{p}^{\alpha}$ is predense in $\mathbb{P}$ with respect to $p$ and $\left|e_{p}^{\alpha}\right|<\kappa$.

- $e_{p}^{\alpha}$ is superdense with respect to $p$ in $\Pi(R)$, where

$$
R=\bigcup\left\{e_{p^{\prime}}^{\beta}: \beta<\alpha \text { and } p^{\prime} \in E^{\beta}\lceil p\} \cup\left\{q_{\beta}: \beta<\alpha\right\} .\right.
$$

- Let $G$ be an internally $\mathbb{P}$ generic filter over $V$. Assume that $\pitchfork_{\lambda,<\kappa}$ and $<\kappa$ covering for subsets of $\lambda$ holds between $V$ and $V[G]$. Then $G \cap E^{\alpha} \neq \emptyset$, for all $\alpha<\mu$. Furthermore, there exists $p^{*} \in G$ such that $\left.\left|E^{\alpha}\right| p^{*}\right|^{V}<\kappa$, for all $\alpha<\mu$.

Before getting started on the construction of the $E^{\alpha}$ 's and $e_{p}^{\alpha}$ 's, let us prove a lemma that lets us improve predense sets to antichains.

Lemma 6.2. Suppose that $P \subseteq \mathbb{Q}$ is a set. There exists an antichain $A \subseteq \mathbb{Q}$ such that

- $|A| \leqslant|P|$

- every condition in $A$ meets $P$; and

- if $p \in P$, then $A$ is predense with respect to $p$.

Proof: Let $\left\langle r_{i}: i<\theta\right\rangle$ enumerate $P$. Define $\left\langle a_{i}: i<\theta^{\prime}\right\rangle$, for some $\theta^{\prime}<\theta^{+}$, by recursion. At stage $i$, if there exists $k<\theta$ such that $r_{k} \sim \bigwedge_{j<i}-a_{j}$, let $k_{i}<\theta$ be least with this property and set $a_{i}=r_{k_{i}} \wedge \bigwedge_{j<i}-a_{j}$. Otherwise, set $\theta^{\prime}=\theta$. Set $A=\left\{a_{i}: i<\theta^{\prime}\right\}$.

By construction $A$ is an antichain and is predense with respect to any condition in $P$. Any condition meeting $A$ meets $P$ because $a_{i} \leqslant r_{k_{i}} \in P$. Finally, $\left|\theta^{\prime}\right| \leqslant \theta$, since $i \mapsto k_{i}$ is a one-to-one function from $\theta^{\prime}$ into $\theta$. (If $j<i$ and $k_{j}=k_{i}$, then $a_{i}=r_{k_{i}} \wedge \bigwedge_{\delta<i}-a_{\delta} \leqslant r_{k_{j}} \wedge \bigwedge_{\delta<j}-a_{\delta}=a_{j}$, which is absurd.

The construction of the $E^{\alpha}$ 's and $e_{p}^{\alpha}$ 's will be by recursion on $\alpha<\mu$. At limit stages in the construction, the following lemma will be useful. This lemma also establishes that, if the construction can be carried out, then there exists $p^{*} \in G$ such that $\left.\left|E^{\alpha}\right| p^{*}\right|^{V}<\kappa$, for all $\alpha<\mu$.

Lemma 6.3. Let $\alpha \leqslant \mu$ be a limit ordinal and assume that $E^{\beta}$ and $e_{p^{\prime}}^{\beta}$ are as required, for $\beta<\alpha$ and $p^{\prime} \in E^{\beta}$. There exists $p \in G$ such that $\mid E^{\beta}\lceil p \mid<\kappa$, for all $\beta<\alpha$.

Proof: Work in $V$. Let $\eta=\operatorname{cf}(\alpha)$ and let $\left\langle\beta_{\delta}: \delta\langle\eta\rangle\right.$ be increasing and cofinal in $\alpha$. Let $\left\langle q_{i}^{\delta}: i<\theta^{\delta}\right\rangle$ be a one-to-one enumeration of $E^{\beta \delta}$, where $\theta^{\delta} \leqslant \lambda$.

In $V[G]$, set

$$
x=\left\{\ll \delta, i »: \delta<\eta \text { and } i<\theta^{\delta} \text { and } q_{i}^{\delta} \in G\right\} .
$$

This set lies in $V[G]$ because $G$ is amenable to $V[G]$. Furthermore, for each $\delta<\eta$, there exists a unique $i$ such that $\ll \delta, i » \in x$. 


\section{ITERATED REDUCTION}

Recall that $<\kappa$-covering for subsets of $\lambda$ holds by hypothesis, thus $\alpha$ has cofinality less than $\kappa$ in $V[G]$ iff it has cofinality less than $\kappa$ in $V$.

If $\boldsymbol{\eta}<\boldsymbol{\kappa}$, then $|x|^{V[G]}=|\eta|^{V[G]}<\kappa$. Using $<\kappa$-covering, let $y \in V$ be a superset of $x$ such that $|y|^{V}<\kappa$.

Working in $V$, set

$$
p=\bigwedge_{\delta<\eta} \bigvee\left\{q_{i}^{\delta}: \ll \delta, i \gg \in y \text { and } i<\theta^{\delta}\right\}
$$

Then $p \in G$. (Otherwise $-p \in G$, contradicting our choice of $x$.) We maintain that $\mid E^{\beta}\left\lceil p \mid<\kappa\right.$, for all $\beta<\alpha$. Fix $\beta<\alpha$ and let $\delta<\eta$ be least such that $\beta_{\delta} \geqslant \beta$. Then

$$
E^{\beta}\left\lceilp \subseteq \bigcup _ { \ll \delta , i \gg \in y } E ^ { \beta } \left\lceil q_{i}^{\delta} .\right.\right.
$$

And $\mid E^{\beta}\left\lceil q_{i}^{\delta} \mid<\kappa\right.$ by hypothesis. Hence $\mid E^{\beta}\lceil p \mid<\kappa$, as required for the lemma.

If $\boldsymbol{\eta} \geqslant \boldsymbol{\kappa}$, then $|x|^{V[G]}=\eta$. By $\pitchfork_{\lambda,<\kappa}$, we have $\pitchfork_{\lambda,<\eta}$, so, by Lemma $3.3 \mathrm{~b}$, we have $\eta$-sequence cocovering for $H \in V[G]$ such that $H: \eta \rightarrow \lambda$. Let $y \in V$ be such that $|y|^{V}=\eta$ and $y \subseteq x$. Set

$$
p=\bigwedge_{\ll \delta, i \gg \in y} q_{i}^{\delta} .
$$

Then $p \in G$, again because $-p \in G$ contradicts our choice of $x$. If $\beta<\alpha$, let $\delta$ be least such that $\beta_{\delta} \geqslant \beta$ and $\ll \delta, i \gg \in y$, for some $i$. Then $E^{\beta}\left\lceil p \subseteq E^{\beta}\left\lceil q_{i}^{\delta}\right.\right.$. Hence $\mid E^{\beta}\lceil p \mid<\kappa$, as required for the lemma.

The construction. Work in $V$. Begin by setting $E^{0}=\{\mathbb{1}\}$ and $e_{\mathbb{1}}^{0}=\{\mathbb{1}\}$.

If $\alpha>0$, we first define an antichain $A \subseteq \mathbb{Q}$, then extend conditions in $A$ to get the antichain $E^{\alpha}$. At the same time, we define $e_{p}^{\alpha}$, for $p \in E^{\alpha}$.

If $\alpha=\beta+1$, set $A=E^{\beta}$.

If $\alpha<\mu$ is a limit ordinal, let

$$
\begin{aligned}
p \in P \quad \text { iff } & p=\bigwedge_{\beta<\alpha} \bigvee x_{\beta} \neq \mathbb{D}, \text { for some }\left\langle x_{\beta}: \beta<\alpha\right\rangle \\
& \text { such that } x_{\beta} \subseteq E^{\beta} \text { and }\left|x_{\beta}\right|<\kappa .
\end{aligned}
$$

Then $|P| \leqslant\left(\lambda^{<\kappa}\right)^{<\mu}=\lambda$, since $\lambda^{<\mu}=\lambda$. By the previous lemma, $P \cap G \neq \emptyset$. Using the Lemma 6.2 , let $A$ be an antichain in $\mathbb{Q}$ such that every condition in $A$ meets $P$, $A$ is predense with respect to each condition in $P$, and $|A| \leqslant|P| \leqslant \lambda$.

Now that we have defined the antichain $A$, we can proceed to define $E^{\alpha}$ and $e_{p}^{\alpha}$, for $p \in E^{\alpha}$. Fix $\bar{p} \in A$ and set

$$
\mathbb{R}=\Pi\left(\bigcup\left\{e_{p^{\prime}}^{\beta}: \beta<\alpha \text { and } p^{\prime} \in E^{\beta}\lceil\bar{p}\} \cup\left\{q_{\beta}: \beta<\alpha\right\}\right) .\right.
$$

Then $\mathbb{R} \subseteq \mathbb{Q}$ and $\mathbb{R}$ is closed under $\Lambda$. Because $\bar{p}$ meets $P$, we know that $\left|E^{\beta}\right| \bar{p} \mid<\kappa$, for all $\beta<\alpha$. By induction, we know that $\left|e_{p^{\prime}}^{\beta}\right|<\kappa$, for $p^{\prime} \in E^{\beta}$. And $\alpha<\mu$. It follows that $|\mathbb{R}| \leqslant 2^{<\mu} \leqslant \lambda^{<\mu}=\lambda$. 


\section{ITERATED REDUCTION}

Define $D_{\bar{p}} \subseteq[\mathbb{R}]^{<\kappa}$ by

$$
D_{\bar{p}}=\left\{d \in[\mathbb{R}]^{<\kappa}: d \text { is superdense in } \mathbb{R} \text { with respect to some } p \in \Pi^{2}(\mathbb{R})\right\} .
$$

For $d \in D_{\bar{p}}$, set

$$
p(d)=\bigvee\left\{p \in \Pi^{2}(\mathbb{R}): d \text { is superdense in } \mathbb{R} \text { with respect to } p\right\} .
$$

Finally, set

$$
P_{\bar{p}}=\left\{p(d): d \in D_{\bar{p}}\right\}
$$

Note that $\left|P_{\bar{p}}\right| \leqslant \lambda$ because $\left|[\mathbb{R}]^{<\kappa}\right| \leqslant \lambda$.

Claim 6.4. If $\bar{p} \in G \cap A$, then $P_{\bar{p}} \cap G \neq \emptyset$.

Proof: Trivially $\mathbb{R}$ is superdense in $\mathbb{R}$ with respect to $\bar{p}$. Set $\tilde{p}=h_{\Pi(\mathbb{R})}(\bar{p})$. Then $\tilde{p} \in G \cap \Pi(\mathbb{R})$. By the Superdensity Reduction Lemma, there exists $p^{*} \in G \cap \Pi^{2}(\mathbb{R})$ and $d \in[\mathbb{R}]^{<\kappa}$ such that $d$ is superdense in $\mathbb{R}$ with respect to $p^{*}$. Then $p^{*} \leqslant p(d) \in P_{\bar{p}}$. So $p(d) \in G \cap P_{\bar{p}}$.

Apply the Lemma 6.2 to get an antichain $A_{\bar{p}} \subseteq \mathbb{Q}$ such that every condition in $A_{\bar{p}}$ meets $P_{\bar{p}}$, and $A_{\bar{p}}$ is predense with respect to any condition in $P_{\bar{p}}$, and $\left|A_{\bar{p}}\right| \leqslant\left|P_{\bar{p}}\right| \leqslant \lambda$.

Note that if $P_{\bar{p}} \cap G \neq \emptyset$, then $A_{\bar{p}} \cap G \neq \emptyset$ because $G$ is internally generic and $A_{\bar{p}}$ is a set of conditions that is predense with respect to any condition in $P_{\bar{p}} \cap G$

For $p \in A_{\bar{p}}$, set $e_{p}^{\alpha}=d$, where $d \in D_{\bar{p}}$ is chosen so that $p \leqslant p(d)$. Then $e_{p}^{\alpha}$ is superdense in $\mathbb{R}$ with respect to $p$.

Finally, set $E^{\alpha}=\bigcup_{\bar{p} \in A} A_{\bar{p}}$. This completes the construction.

\section{The factor lemma}

Let us continue to work with the following

\section{Assumptions.}

- $\mathbb{P}$ is either an element of $V$ or a $V$-definable partial ordering that is separative and is closed under finite meets.

- $\mathbb{Q} \subseteq \mathbb{P}$ is, in $V$, a set-complete Boolean algebra with $\mathbb{0}$ removed, and $\mathbb{Q}$ is complete in $\mathbb{P}$.

- $G$ is a filter on $\mathbb{P}$ that is internally generic over $V$.

Working in $V$, if $Q \subseteq \mathbb{Q}$, let $\mathbb{B}(Q)$ be the set-complete (in $\mathbb{Q}$ ) Boolean subalgebra generated by $Q$. In general, no set in $V$ is dense in $\mathbb{B}(Q)$, even if $Q$ is a set in $V$. If $p \in \mathbb{P}$, let

$$
\mathbb{B}_{p}(Q)=\{p \wedge q: q \in \mathbb{B}(Q) \text { and } p \wedge q \neq 0\} .
$$

Then $\mathbb{B}_{p}(Q)$ is the smallest subclass of $\mathbb{Q}$ that contains $\{p \wedge q: q \in Q\}$ and is closed under $\bigwedge$ and complements relative to $p$. That is, if $q \in \mathbb{B}_{p}(Q)$ and $p \wedge-q \neq \mathbb{0}$, then $p \wedge-q \in \mathbb{B}_{p}(Q)$. 


\section{THE FACTOR LEMMA}

Factor Lemma 7.1. In $V$, let $\kappa, \mu$, and $\lambda$ be cardinals such that

- $\omega_{1} \leqslant \kappa<\mu \leqslant \lambda$,

- $\kappa$ is regular,

- $\operatorname{cf}(\mu)>\kappa$, and

- $\lambda^{<\mu}=\lambda$.

Let $Q \subseteq \mathbb{Q}$ have cardinality at most $\mu$. Assume that

- $<\kappa$-covering for subsets of $\lambda$ and

- $\pitchfork_{\lambda,<\kappa}$

hold between $V$ and $V[G]$. There exists $p^{*} \in G$ such that $\mathbb{B}_{p^{*}}(Q)$ has a dense subset of cardinality less than $\kappa$.

Proof: Let $\left\langle q_{\alpha}: \alpha<\mu\right\rangle$ be an enumeration of $Q$ in $V$. Let $E^{\alpha}, e_{p}^{\alpha}$, and $p^{*} \in G$ be as provided by the Iterated Superdensity Reduction Lemma.

Note first that there exists $p \in G$ such that $E^{\alpha}$ is predense with respect to $p$, for all $\alpha<\mu$ : Because $G$ is internally generic, it contains a condition $p$ deciding $d=\left\{-\bigvee E^{\alpha}: \alpha<\mu\right\}$. But $G \cap d=\emptyset$, since $G \cap E^{\alpha} \neq \emptyset$, for all $\alpha$. Let $p \in G$ be incompatible with every element of $d$. Then $p \leqslant-\bigvee d=\bigwedge_{\alpha<\mu} \bigvee E^{\alpha}$.

Assume, then, that the condition $p^{*} \in G$ provided by Iterated Superdensity Reduction has the property that $E^{\alpha}$ is predense with respect to $p^{*}$, for all $\alpha \leqslant \mu$. Set

$$
\begin{aligned}
d_{\alpha} & =\bigcup_{p \in E^{\alpha} \nmid p^{*}} e_{p}^{\alpha} ; \\
R_{\alpha} & =\bigcup_{\beta<\alpha} d_{\beta} \cup\left\{q_{\beta}: \beta<\alpha\right\} ; \\
\mathbb{R}_{\alpha} & =\Pi\left(R_{\alpha}\right) ; \\
\mathbb{R}_{<\alpha} & =\bigcup_{\beta<\alpha} \mathbb{R}_{\beta} ; \\
\mathbb{R}_{\alpha}^{*} & =\left\{p^{*} \wedge r: r \in \mathbb{R}_{\alpha}\right\} ; \text { and } \\
\mathbb{R}_{<\alpha}^{*} & =\bigcup_{\beta<\alpha} \mathbb{R}_{\beta}^{*} .
\end{aligned}
$$

Note that

(1) If $\alpha<\mu$, then $d_{\alpha}$ is superdense in $\mathbb{R}_{\alpha}$ with respect to $p^{*}$. This is because $E^{\alpha}$ is predense with respect to $p^{*}$ and $e_{p}^{\alpha}$ is superdense in $\mathbb{R}_{\alpha}$ with respect to $p$.

(2) Suppose that $\alpha \leqslant \alpha^{\prime}<\mu$. If $q \in d_{\alpha}$ is compatible with $p^{*}$, then there exists $q^{\prime} \in d_{\alpha^{\prime}}$ such that $q^{\prime} \leqslant q$, since $d_{\alpha^{\prime}}$ is superdense in $\mathbb{R}_{\alpha^{\prime}}$ with respect to $p^{*}$.

(3) $\left|d_{\alpha}\right|<\kappa$

(4) If $\alpha \leqslant \beta$, then $\mathbb{R}_{\alpha} \subseteq \mathbb{R}_{\beta}$. Also $\mathbb{R}_{<\alpha} \subseteq \mathbb{R}_{\alpha}$.

To render our notation less cumbersome, let $h_{\alpha}=h_{\mathbb{R}_{\alpha}}$, that is, for $p \in \mathbb{P}$, set

$$
h_{\alpha}(p)=\bigwedge\left\{r \in \mathbb{R}_{\alpha}: p \leqslant r\right\} .
$$

Claim 7.2. If $\alpha \leqslant \mu$ is a limit ordinal, then $\mathbb{R}_{<\alpha}^{*}$ is closed under complements relative to $p^{*}$. That is, if $r \in \mathbb{R}_{<\alpha}$ and $p^{*} \wedge-r \neq \mathbb{0}$, then $p^{*} \wedge-r \in \mathbb{R}_{<\alpha}^{*}$.

Proof: Suppose that $r \in \mathbb{R}_{\beta}$, where $\beta<\alpha$. It suffices to see that

$$
p^{*} \wedge-r=p^{*} \wedge \wedge\left\{-q: q \leqslant r \text { and } q \in d_{\beta+1}\right\}
$$


because $\bigwedge\left\{-q: q \leqslant r\right.$ and $\left.q \in d_{\beta+1}\right\} \in \Pi\left(R_{\beta+2}\right)=\mathbb{R}_{\beta+2} \subseteq \mathbb{R}_{<\alpha}$.

On the one hand, if $q \leqslant r$, then $-r \leqslant-q$. Hence

So $(*)$ holds with " $\leqslant$ " in place of " $=$ ".

$$
-r \leqslant \bigwedge\left\{-q: q \leqslant r \text { and } q \in d_{\beta+1}\right\} .
$$

For the converse relation, suppose for a contradiction that

$$
p^{*} \wedge \wedge\left\{-q: q \leqslant r \text { and } q \in d_{\beta+1}\right\} \not-r .
$$

Then there exists $p$ such that

$$
p \leqslant p^{*} \wedge r \wedge \wedge\left\{-q: q \leqslant r \text { and } q \in d_{\beta+1}\right\} .
$$

Now $d_{\beta+1}$ is superdense in $\mathbb{R}_{\beta+1}$ with respect to $p^{*}$, so there exists $q \in d_{\beta+1}$ be such that $q \sim p$ and $q \leqslant h_{\beta+1}(p)$. Then $q \leqslant r$ because $p \leqslant r \in \mathbb{R}_{\beta}$. But then $p \leqslant-q$ by (**), contradicting that $q \sim p$, and completing the proof of the Claim.

Claim 7.3. If $\alpha \leqslant \mu$ is a limit ordinal and $q \in \mathbb{R}_{\alpha}$, then $q=\bigwedge_{\beta<\alpha} h_{\beta}(q)$.

Proof: Since $h_{\beta}(q) \geqslant q$, it is evident that $q \leqslant \bigwedge_{\beta<\alpha} h_{\beta}(q)$. For the converse relation, let $x, y \subseteq R_{\alpha}$ be such that

$$
q=\bigwedge_{r \in x}-r \wedge \bigwedge_{r \in y} r .
$$

If $r \in R_{\alpha}$, then $r \in R_{\beta}$, for some $\beta<\alpha$. Then $-r \in \Pi\left(R_{\beta}\right)=\mathbb{R}_{\beta}$, provided $-r \neq \mathbb{0}$. Thus

- if $r \in y$, then $h_{\beta}(q) \leqslant r$, for all sufficiently large $\beta<\alpha$, and

- if $r \in x$, then $h_{\beta}(q) \leqslant-r$, for all sufficiently large $\beta<\alpha$.

Hence $\bigwedge_{\beta<\alpha} h_{\beta}(q) \leqslant \bigwedge_{r \in x}-r \wedge \bigwedge_{r \in y} r=q$.

Our goal is to see that $\mathbb{R}_{<\mu}^{*}$ has a dense subset of cardinality less than $\kappa$. On the road to that conclusion, let us prove

\section{Claim 7.4.}

(a) $\mathbb{R}_{<\mu}^{*}$ is $<\kappa$-c.c.

(b) $\mathbb{R}_{\alpha}^{*}$ is $<\kappa$-c.c., for all $\alpha<\mu$.

Proof: Note first that (b) follows from (a): If $\alpha<\mu$, then $\mathbb{R}_{\alpha}^{*}$ is closed under $\Lambda$. Thus two conditions in $\mathbb{R}_{\alpha}^{*}$ are compatible in $\mathbb{R}_{\alpha}^{*}$ iff they are compatible in $\mathbb{R}_{\beta}^{*}$, for all $\beta \geqslant \alpha$. Consequently an antichain of cardinality $\kappa$ in $\mathbb{R}_{\alpha}^{*}$ would be an antichain in $\mathbb{R}_{<\mu}^{*}$, contradicting part (a).

To prove part (a), suppose that $A^{*} \subseteq \mathbb{R}_{<\mu}^{*}$ is an antichain (perhaps not maximal) and $\left|A^{*}\right| \leqslant \kappa$. Our goal is to see that $\left|A^{*}\right|<\kappa$. Let $A \subseteq \mathbb{R}_{<\mu}$ be such that $A^{*}=$ $\left\{p^{*} \wedge r: r \in A\right\}$ and $|A| \leqslant \kappa$ (though $A$ may not be an antichain). Choose $\alpha<\mu$ large enough that $A \subseteq \mathbb{R}_{\alpha}$.

Fix $a \in A^{*}$. Then $a \leqslant p^{*}$. Because $d_{\alpha}$ is superdense with respect to $p^{*}$, there exists $q \in d_{\alpha}$ such that $q \sim a$ and $q \leqslant h_{\alpha}(a)$. Then $q \wedge p^{*} \neq \mathbb{0}$ because $q \sim a \leqslant p^{*}$. Let $r \in A$ be such that $a=p^{*} \wedge r$. Then $q \leqslant r$, since $q \leqslant h_{\alpha}(a)$. So $p^{*} \wedge q \leqslant p^{*} \wedge r$.

Because $a \in A^{*}$ was arbitrary, this shows that

$$
\forall a \in A^{*} \exists q \in d_{\alpha}\left(p^{*} \wedge q \neq \mathbb{0} \text { and } p^{*} \wedge q \leqslant a\right) .
$$

Because $\left|d_{\alpha}\right|<\kappa$ and $A^{*}$ is an antichain, this shows that $\left|A^{*}\right|<\kappa$, completing the proof of the Claim. 
Recall that $d_{\alpha}=\bigcup_{p \in E^{\alpha} \uparrow p^{*}} e_{p}^{\alpha}$. Set

$$
d_{\alpha}^{*}=\left\{p^{*} \wedge r: r \in d_{\alpha} \text { and } p^{*} \sim r\right\}
$$

Claim 7.5.

(a) If $\alpha<\mu$ is a limit ordinal and $\operatorname{cf}(\alpha) \geqslant \kappa$, then there exists $\beta<\alpha$ such that $d_{\beta}^{*}$ is dense in $\mathbb{R}_{\alpha}^{*}$.

(b) There exists $\beta<\mu$ such that $d_{\beta}^{*}$ is dense in $\mathbb{R}_{<\mu}^{*}$.

Proof: First note that part (b) follows from part (a): Assume part (a). Recall that $\operatorname{cf}(\mu)>\kappa$. By Fodor's Lemma, there exists $\beta<\mu$ such that $d_{\beta}^{*}$ is dense in $\mathbb{R}_{\alpha}^{*}$ for $\alpha$ unbounded in $\mu$. But $\mathbb{R}_{<\mu}^{*}=\bigcup_{\alpha<\mu} \mathbb{R}_{\alpha}^{*}$ and $\mathbb{R}_{\alpha}^{*} \subseteq \mathbb{R}_{\beta}^{*}$, for $\alpha \leqslant \beta$.

Turning to the proof of part (a), begin by noting that it suffices to show that

$$
\text { there exists } \beta<\alpha \text { such that each element of } d_{\alpha}^{*} \text { is extended by some }
$$
element of $d_{\beta}^{*}$.

To see that $(*)$ suffices, suppose $r \in \mathbb{R}_{\alpha}$ is compatible with $p^{*}$. We must find $q^{\prime} \in d_{\beta}$ such that $p^{*} \wedge q^{\prime} \leqslant p^{*} \wedge r$. Let $q \in d_{\alpha}$ be such that $q \leqslant h_{\alpha}\left(p^{*} \wedge r\right)$ and $q \sim p^{*} \wedge r$. Then $q \leqslant r$, since $r \in \mathbb{R}_{\alpha}$. And $p^{*} \wedge q \in d_{\alpha}^{*}$. Let $q^{\prime} \in d_{\beta}$ be such that $p^{*} \wedge q^{\prime} \leqslant p^{*} \wedge q$. Then $p^{*} \wedge q^{\prime} \leqslant p^{*} \wedge r$.

For a contradiction, suppose that $(*)$ fails. Then

$$
\forall \beta<\alpha \exists q \in d_{\alpha} \forall r \in d_{\beta}\left(p^{*} \wedge r \notin p^{*} \wedge q\right) .
$$

Because $\left|d_{\alpha}\right|<\kappa$ and $\operatorname{cf}(\alpha) \geqslant \kappa$, it follows that there exists $q \in d_{\alpha}$ such that for $\beta$ unbounded in $\alpha$, no condition in $d_{\beta}^{*}$ extends $q$. Because every condition in $d_{\beta}$ is extended by some condition in $d_{\beta^{\prime}}$ when $\beta^{\prime} \geqslant \beta$, it follows that

$$
\forall \beta<\alpha \forall r \in d_{\beta}\left(p^{*} \wedge r \not p^{*} \wedge q\right) .
$$

Now $q=\bigwedge_{\gamma<\alpha} h_{\gamma}(q)$. Thus

$$
\forall \beta<\alpha \forall r \in d_{\beta} \exists \gamma<\alpha\left(p^{*} \wedge r \not p^{*} \wedge h_{\gamma}(q)\right) .
$$

Let $\left\langle\gamma_{i}: i<\kappa\right\rangle$ be an increasing sequence of ordinals less than $\alpha$ such that for $i<$ $j<\kappa$,

$$
\forall r \in d_{\gamma_{i}}\left(p^{*} \wedge r \notin p^{*} \wedge h_{\gamma_{j}}(q)\right) .
$$

Using that $d_{\gamma_{i}}$ is superdense in $\mathbb{R}_{\gamma_{i}}$ with respect to $p^{*}$, let $r_{i} \in d_{\gamma_{i}}$ be such that $r_{i} \sim p^{*} \wedge q$ and $r_{i} \leqslant h_{\gamma_{i}}\left(p^{*} \wedge q\right)$. Then $r_{i} \wedge-h_{\gamma_{i+1}}(q) \sim p^{*}$ by $(\dagger)$. Set

$$
q_{i}=p^{*} \wedge r_{i} \wedge-h_{\gamma_{i+1}}(q) .
$$

If $i<j<\kappa$, then $q_{j} \leqslant r_{j} \leqslant h_{\gamma_{j}}(q)$, but $q_{i} \leqslant-h_{\gamma_{i+1}}(q) \leqslant-h_{\gamma_{j}}(q)$. Thus $A^{*}=$ $\left\{q_{i}: i<\kappa\right\}$ is an antichain in $\mathbb{R}_{\alpha}^{*}$, contradicting the previous Claim and completing the proof of the current one. 


\section{THE FACTOR LEMMA}

Trivially $\mathbb{R}_{\alpha}^{*}$ is closed under $\Lambda$, for all $\alpha$. We have seen that if $\alpha$ is a limit ordinal, then $\mathbb{R}_{<\alpha}^{*}$ is closed under complements relative to $p^{*}$. We can now show

Claim 7.6. If $\alpha \leqslant \mu$ and $\operatorname{cf}(\alpha) \geqslant \kappa$, then $\mathbb{R}_{<\alpha}^{*}$ is closed under $\bigwedge$.

Proof: If $x \subseteq \mathbb{R}_{<\alpha}^{*}$ and $\bigwedge x \neq \mathbb{0}$, then $\bigwedge x \in \mathbb{R}_{\alpha}^{*}$. Let $\beta<\alpha$ be such that $d_{\beta}^{*}$ is dense in $\mathbb{R}_{\alpha}^{*}$. Set

$$
q=\bigvee\left\{r \in d_{\beta}^{*}: r \leqslant \bigwedge x\right\}
$$

Then $q \in \mathbb{R}_{<\alpha}^{*}$. Certainly $q \leqslant \bigwedge x$. To see $\bigwedge x \leqslant q$, for a contradiction suppose that $p=-q \wedge \wedge x \neq 0$. Now $p \leqslant p^{*}$, so $p^{*} \wedge h_{\alpha}(p) \in \mathbb{R}_{\alpha}^{*}$. Also $h_{\alpha}(p) \leqslant-q \wedge \wedge x$, since $x \subseteq \mathbb{R}_{<\alpha}^{*}$ and $q \in \mathbb{R}_{<\alpha}^{*}$. Let $r \in d_{\beta}^{*}$ be such that $r \leqslant p^{*} \wedge h_{\alpha}(p)$. Then $r \leqslant \bigwedge x$, hence $r \leqslant q$ by $(*)$. But also $r \leqslant-q$. This contradiction completes the proof of Claim 7.6.

We are now ready to finish the proof of the Set Forcing Lemma. By definition, $\mathbb{B}(Q)$ is the smallest subclass of $\mathbb{Q}$ that contains $Q$ and is closed under $\Lambda$ and non- $\mathbb{O}$ complements. Thus $\mathbb{R}_{<\mu} \subseteq \mathbb{B}(Q)$; hence $\mathbb{R}_{<\mu}^{*} \subseteq \mathbb{B}_{p^{*}}(Q)$.

For the converse inclusion, recall that $\mathbb{B}_{p^{*}}(Q)$ is the smallest subclass of $\mathbb{Q}$ that contains $\left\{p^{*} \wedge q: q \in Q\right\}$ and is closed under $\bigwedge$ and complements relative to $p^{*}$. By construction $\left\{p^{*} \wedge q: q \in Q\right\} \subseteq \mathbb{R}_{<\mu}^{*}$ and we have seen $\mathbb{R}_{<\mu}^{*}$ has these two properties.

Thus $\mathbb{B}_{p^{*}}(Q)=\mathbb{R}_{<\mu}^{*}$. There exists $\beta<\mu$ such that $d_{\beta}^{*}$ is dense in $\mathbb{R}_{<\mu}^{*}$. And $\left|d_{\beta}^{*}\right|<\kappa$.

\section{The set forcing theorem}

Set Forcing Theorem 8.1. Let $W$ be an outer model of $V$ and assume that $(W ; V)$ satisfies ZFC. In $V$, let $\kappa, \mu$, and $\lambda$ be cardinals such that

- $\omega_{1} \leqslant \kappa<\mu \leqslant \lambda$,

- $\kappa$ is regular,

- $\operatorname{cf}(\mu)>\kappa$, and

- $\lambda^{<\mu}=\lambda$.

Assume that

- $<\kappa$-covering for subsets of $\lambda$ and

- $\pitchfork_{\lambda,<\kappa}$

hold between $V$ and $W$. Then every subset of $\mu$ in $W$ is set generic over $V$ for a forcing of $V$-cardinality less than $\kappa$.

Proof: The theorem follows from the Factor Lemma once we have represented $W$ as an internally class generic extension of $V$. This is more or less folklore and has appeared elsewhere in various forms. We include a proof here because we need to take advantage of a detail.

Because it is just as easy, we give a proof that applies even to $W$ and $V$ that do not satisfy Choice. This requires the additional assumption that every set in $W$ is the range of a function in $W$ with domain in $V$. This extra hypothesis is trivial if $W$ satisfies Choice. However, it is necessary: If $W=V[G]$ and $\stackrel{\circ}{a}^{G} \in W$, then $f: \operatorname{dom}(\stackrel{\circ}{a}) \rightarrow \stackrel{\circ}{a}^{G}$ given by $f(\stackrel{\circ}{b})=\stackrel{\circ}{b}^{G}$ is a function onto $\stackrel{\circ}{a}^{G}$ with domain in $V$. 


\section{THE SET FORCING THEOREM}

Lemma 8.2. Let $W \supseteq V$ be a countable and standard with $V \cap \mathrm{OR}=W \cap \mathrm{OR}$. Assume that both $V$ and $(W ; V)$ satisfy ZF. Assume also that every set in $W$ is the range of a function in $W$ with domain in $V$. There exists a $V$-definable partial ordering $\mathbb{Q}$ and a (W-amenable) filter $G$ such that $G$ is internally $\mathbb{Q}$-generic over $V$ and $W=V[G]$. Furthermore, $(V[G] ; V, \mathbb{P}, G) \vDash \mathrm{ZF}$ and, in $V, \mathbb{Q}$ is a set-complete Boolean algebra with 0 removed.

Proof: Using a simple class forcing definable over $W$, construct a $W$-amenable class function $F: V \rightarrow W$ such that

(1) $\operatorname{rng}(F)=W$;

(2) if $a \in F(b)$, then $a=F(c)$, for some $c \in b$; and

(3) $(W ; V, F) \vDash \mathrm{ZF}$.

Ordered by reverse functional extension, conditions in this forcing are functions $f \in W$ such that

(a) $\operatorname{dom}(f) \in V$,

(b) $\operatorname{rng}(f)$ is transitive, and

(c) $f$ satisfies requirement (2).

These conditions are $<\infty$-closed. It is easy to check that, given $a \in W$, conditions $f$ with $a \subseteq \operatorname{rng}(f)$ are dense. Let $F$ be given by a filter sufficiently generic to insure property (c).

For future reference, note that, given any $f$ as in (a)-(c), we may assume that $f \subseteq F$, by choosing the generic $F$ below the condition $f$.

Let $\mathcal{L}$ be the language with the binary relation symbol $\in$ and a constant symbol $\dot{a}$, for each $a \in V$. Work in the smallest infinitary fragment (with equality) including all Boolean combinations of atomic formulas of $\mathcal{L}$ that lie in $V$ and contain only finitely many distinct variables. Let $T$ consist of the axioms

$$
\forall x\left(x \in \dot{a} \rightarrow \bigvee_{b \in a} x=\dot{b}\right),
$$

for $a \in V$. Letting $\widehat{W}=(W ; F(a))_{a \in V}$, we have that $\widehat{W} \vDash T$.

Working in $V$, let $\mathbb{Q}$ consist of all quantifier-free infinitary sentences $q$ of $\mathcal{L}$ such that $T \cup\{q\}$ is consistent. Order $\mathbb{Q}$ by $p \geqslant q$ iff $T \vdash q \rightarrow p$. Modded out by the equivalence relation " $p \leqslant q$ and $q \leqslant p$ ", $\mathbb{Q}$ is a set-complete Boolean algebra with $\mathbb{0}$ removed.

Working in $(W ; V, F)$, set $G=\{q \in \mathbb{Q}: \widehat{W} \vDash q\}$. Then $G$ is a filter on $\mathbb{Q}$ and is internally generic: If $d \in V$ is predense then $T \vdash \bigvee d$, so $\widehat{W} \vDash \bigvee d$. Hence $G \cap d \neq \emptyset$.

Clearly $V[G] \subseteq W$ because $G$ is definable over $(W ; V, F)$. Conversely, in $V$ define a Shoenfield term $\stackrel{a}{a}$ for each $a \in V$ by

$$
\stackrel{\circ}{a}=\{(\stackrel{\circ}{b},\{\dot{b} \in \dot{a}\}): b \in a\} .
$$

Then $\stackrel{\circ}{a}^{G}=F(a)$. Hence $W \subseteq V[G]$. This completes the proof of the lemma. 


\section{THE SET FORCING THEOREM}

Returning to the proof of the theorem, suppose that $a \in W$ is a subset of $\mu$. Let $F$ in the proof of the previous lemma be chosen so that $f \subseteq F$, where $f: \mu+1 \rightarrow W$ by $f\lceil\mu=\mathrm{id}\lceil\mu$ and $f(\mu)=a$. Let $G$ be the internally $\mathbb{Q}$-generic filter provided by $F$. In $V$, set

$$
Q=\{\{\dot{\alpha} \in \dot{\mu}\}: \alpha<\mu\} .
$$

Then $Q \subseteq \mathbb{Q}$ is a set of $\mu$ conditions. By the Factor Lemma, there exists $p^{*} \in G$ such that, in $V, \mathbb{B}_{p^{*}}(Q)$ has a dense subset $\mathbb{D}$ of cardinality less than $\kappa$. Then $G \cap \mathbb{D}$ is $\mathbb{D}$-generic over $V$ and $a=F(a)=\stackrel{\circ}{\mu}^{G} \in V[G \cap \mathbb{D}]$.

\section{References}

[B] L. Bukovský, Characterization of generic extensions of models of set theory, Fund. Math. 83 (no. 1) (1973), pp. 35-46.

[J] T. Jech, Set Theory, Third Millennium Edition, Monographs in mathematics, Springer (2002).

[S] M. Stanley, Outer models and genericity, Journal of Symbolic Logic 68(2) (June 2003), pp. 389-418.

Math Department
San Jose State
San Jose, CA 95192
e-mail: stanley@math.sjsu.edu 